ISSNe: 2215-7484

Gestión territorial y medio ambiente

\title{
Manejo de la avifauna como parte de la gestión del arbolado urbano en Bogotá D.C.
}

Handling of Avifauna as Part of the Management of Urban Trees in Bogota, D. C.

Manejo da avifauna como parte da gestão da floresta urbana em Bogotá D. C.

Germán Tovar ${ }^{*}$

Recibido: 15 de noviembre de 2017

Aprobado: 25 de julio de 2018

Doi: http://dx.doi.org/10.12804/revistas.urosario.edu.co/territorios/a.6253

Para citar este artículo:

Tovar, G. (2019). Manejo de la avifauna como parte de la gestión del arbolado urbano en Bogotá D.C. Territorios (40), 83-117. Doi: http://dx.doi.org/10.12804/revistas.urosario.edu.co/territorios/a.6253

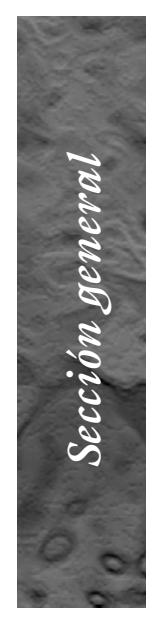

* Ingeniero Forestal, Universidad Distrital "Francisco José de Caldas", Bogotá. Especialista en Administración y Planificación del Desarrollo Regional, Centro de Estudios Interdisciplinarios (CIDER), Universidad de los Andes, Bogotá. Magíster en Gestión Ambiental, Pontifica Universidad Javeriana. ORCID: 0000-0002-3388-3650 
Palabras clave

Arbolado urbano, aves, avifauna urbana, ecología urbana, nidos, riqueza de especies, servicios ecosistémicos.

Keywords

Urban tree, birds, urban avifauna, urban ecology, nests, species richness, ecosystem services.

Palavras-chave

Floresta urbana, aves, avifauna urbana, ecologia urbana, ninhos, riqueza de espécies, serviços ecossistêmicos.

\section{territarias 40}

RESUMEN

El proceso de urbanización de Bogotá impacta directamente sobre la cobertura arbórea, debido a que los proyectos de construcción priorizan la tala en vez del traslado, y sus proyectos paisajísticos compensan insuficientemente su impacto negativo. La reducción progresiva de espacios verdes y arbolado disminuyen los hábitats necesarios para las aves. En la gestión del arbolado urbano, que incluye actividades de plantación, poda, traslado y tala, la ciudad aplica procedimientos para reducir su impacto sobre la avifauna. Su autoridad ambiental ha generado información para ampliar el conocimiento y permitir el entendimiento de la dinámica existente entre árboles y aves urbanas, que sirve de base para analizar la eficiencia de su acción, tanto en el procedimiento de manejo de avifauna como en el diseño y selección de especies en nuevas arborizaciones. Se presentan estadísticas de informes finales de manejo de avifauna de varias obras de infraestructura y los resultados de un estudio elaborado entre la Secretaría Distrital de Ambiente (SDA) y la Asociación Bogotana de Ornitología (ABO), en el que se formulan criterios técnicos para la conectividad ecológica con énfasis en la conservación de aves y la consolidación de procesos de restauración en la Estructura Ecológica Principal de la ciudad.

\section{ABSTRACT}

The urbanization process of Bogota has a direct impact on tree cover, because construction projects prioritize logging instead of transplant, and their landscape projects do not generate sufficient compensation. The progressive reduction of green spaces and trees, diminish the necessary habitats for the birds. In the management of the urban trees that includes activities of planting, pruning, transplant and logging, the city applies procedures to reduce its impact on avifauna. Its environmental authority has generated information to expand knowledge and allow understanding of the dynamics between trees and urban birds, which serves as a basis to analyze the efficiency of its action, both in the birdlife management procedure and in the design and selection of species in new tree plantings. This article shows statistics from a review of avifauna management final reports in various infrastructure projects and the work's results of a study prepared between the District Department of Environment -SDA- and the Bogota's Ornithology Association -ABO-, in which technical criteria are formulated for the ecological connectivity with emphasis on bird conservation and consolidation of restoration processes in the city's main ecological structure.

\section{RESUMO}

O processo de urbanização de Bogotá impacta diretamente sobre a cobertura arbórea, devido a que os projetos de construção, priorizam a tala em lugar do traslado, e seus projetos paisagísticos compensam insuficientemente seu impacto negativo. A redução progressiva de espaços verde e floresta, diminuem os hábitats necessários para as aves. Na gestão da floresta urbana, que inclui atividades de plantação, poda, traslado e tala; a cidade aplica procedimentos para reduzir seu impacto sobre a avifauna. Sua autoridade ambiental tem gerado informação para ampliar o conhecimento e permitir o entendimento da dinâmica existente entre árvores e aves urbanas, 
que serve de base para analisar a eficiência de sua ação, tanto no procedimento de manejo de avifauna quanto no desenho e seleção de espécies em novas arborizações. Se apresentam estatísticas de informes finais de manejo de avifauna de várias obras de infraestrutura e os resultados de um estudo elaborado entre a Secretaria Distrital de Ambiente -SDA- e a Associação Bogotana de Ornitologia $-\mathrm{ABO}-$, no que se formulam critérios técnicos para a conectividade ecológica com ênfase na conservação de aves e a consolidação de processos de restauração na Estrutura Ecológica Principal da cidade.

\section{Introducción}

Bogotá, capital de Colombia, es habitada por cerca de 8 millones de personas, asentadas sobre una llanura con un promedio de $2630 \mathrm{~m}$ de altitud, constituyéndose como la metrópoli más alta del mundo. Está bordeada por una cadena montañosa que alcanza los $3250 \mathrm{~m}$ de altitud. Tiene un área total de $1776 \mathrm{~km}^{2}$ y un área urbana de $384,3 \mathrm{~km}^{2}$. Se sitúa sobre lo que fue antiguamente un lago, del cual dan evidencia los 16 humedales que, actuando como parches, cubren algunos sectores del área urbana con una superficie de 719 hectáreas. Tiene un clima templado de altura cuyas temperaturas regularmente oscilan entre los $6^{\circ} \mathrm{C}$ y $24^{\circ} \mathrm{C}$, con una media anual de $15^{\circ} \mathrm{C}$. Se caracteriza, además, por tener un amplio sistema de parques que se integran entre sí por un conjunto de corredores peatonales y ciclorutas. Estos se enlazan con las zonas verdes, plazoletas, plazas, humedales, alamedas, áreas protegidas y áreas de manejo especial, lo que configura la denominada Estructura Ecológica Principal (EEP), concepto que internacionalmente es similar al de infraestructura verde, según la definición de la Unión Europea (2013).
El Plan de Ordenamiento Territorial de Bogotá (Decreto No 190, 2004) define la EEP en el Artículo 17 como una red de espacios y corredores que sostienen y conducen la biodiversidad y los procesos ecológicos esenciales, por medio del territorio de la ciudad, en sus diferentes formas e intensidades de ocupación, lo que lo dota de servicios ambientales para su desarrollo sostenible. Tiene como base la estructura ecológica, geomorfológica y biológica original: los cerros, el valle aluvial del río Bogotá y la planicie, complementándose por medio del conjunto de reservas, parques y remanentes de la vegetación natural asociada con quebradas y ríos presentes en la ciudad. Según Remolina (2006), uno de los principios para que la red funcione ecológicamente es mantener la conectividad estructural entre los elementos de la EEP.

El proceso de urbanización tiene un impacto directo sobre la cobertura arbórea, debido a que los proyectos de construcción priorizan la tala de los especímenes forestales en lugar de su traslado, y sus propuestas paisajísticas no generan una compensación suficiente en lo ambiental. Para McCallum y Dobson (2002) y Adams (2005), la urbanización fragmenta el paisaje, lo que 
destruye o modifica el hábitat de muchas especies locales, rompe procesos ecológicos y crea nuevos hábitats para especies con alta capacidad de tolerancia al disturbio.

El endurecimiento progresivo de las áreas verdes de Bogotá se impulsa principalmente por medio de tres normas: el Decreto 561 de 2015, denominado Decreto de andenes, mediante el cual se elimina la posibilidad de coexistencia de la zona dura de andén con zonas empradizadas; la Resolución SDA-SDP 456 de 2014, que reglamenta el Acuerdo Distrital 327 de 2008, que permite el endurecimiento de área verde por razón de la construcción de obra pública, aun cuando exige su compensación en área verde efectiva dentro del área de influencia del proyecto, y la Resolución SDA-SDP 73 de 2017, que modifica la anterior restringiendo la obligación de realizar dicha compensación.

La urbanización afecta a las aves en todo el mundo y produce homogeneidad, alta densidad y accesibilidad de especies exóticas dentro de los asentamientos humanos (Erz, 1966; Bezzel, 1985; Knigtht, 1990; Marzluff et al., 1998; Blair, 2001 \& Marzluff, 2001). Así mismo, la perturbación es manifiesta debido a que se facilita la acción de los depredadores naturales; atrae nuevos predadores, enfermedades y competidores; afecta la estructura de la cadena trófica al eliminar los depredadores de nivel superior; obstruye la dispersión de individuos; elimina recursos clave como árboles y arbustos dispuestos en corredores o parches; y altera el ciclo de nutrientes y del agua (Marzluff \& Ewing, 2001).
Para Donnelly y Marzluff (2004), se deben realizar estudios que diluciden cómo el patrón del asentamiento humano y las propiedades de las reservas afectan varios factores limitantes importantes como: depredación de nidos, disponibilidad de alimento y disponibilidad de sitios para anidar. La comprensión de cómo la condición local y del nivel del paisaje afecta los factores limitantes permite diseñar maneras de reducir los efectos sobre las poblaciones. Por tanto, es importante complementar la medición directa de la reproducción y la supervivencia de individuos en paisajes urbanos, con múltiples medidas indirectas como la proporción de juveniles a adultos, tasas de depredación, parasitismo y fuentes de alimento.

Los bogotanos, además de expresar su preocupación por la subsistencia de los árboles, manifiestan también su desazón por el desplazamiento de las aves. Según Delgado y Correa (2013), aun cuando algunas de las principales ciudades del país cuentan con un estimativo de su diversidad actual, la ornitología urbana colombiana es todavía un área joven que debe articularse con los ámbitos sociales, culturales, políticos y económicos de las ciudades.

Acogiendo el postulado de Müller y Werner (2010), respecto a que las ciudades deben propender por asegurar el uso sustentable de bienes y servicios ambientales y la gestión para conservar la biodiversidad, Bogotá, por medio de su autoridad ambiental (SDA), ha sido consecuente con la necesidad de establecer parámetros de manejo para la avifauna, cuando se interviene 
la cobertura arbórea durante los procesos de construcción de obras públicas, $y$, por ello, mediante la Resolución 991 de 2001, incluyó procesos y procedimientos para su manejo, en la Guía de Manejo Ambiental para el desarrollo de proyectos de infraestructura urbana en Bogotá D.C., del Instituto de Desarrollo Urbano, entidad del gobierno municipal responsable de la construcción de espacio público. La práctica de esos procedimientos ha propiciado el hallazgo de nidos, huevos y polluelos, cuyos resultados se revelan en el desarrollo de este artículo.

En la paulatina reducción de los espacios verdes y el arbolado de la ciudad, algunos sectores de la población advierten sobre la contracción de los hábitats necesarios para la existencia de la avifauna bogotana. Por ello, es necesario dimensionar el impacto que la intervención del arbolado por construcción de obras públicas de gran tamaño ha causado sobre la avifauna, en términos de anidación y supervivencia. Además, por medio del análisis de áreas del paisaje actual y de la distribución y abundancia de especies tanto arbóreas como de aves, se acomete la descripción del impacto que el urbanismo ha ocasionado respecto del deterioro del hábitat y la alteración en la presencia de especies de avifauna en el área urbana de Bogotá. Finalmente, se presenta una aproximación al entendimiento de la dinámica que existe entre árboles y aves, en el entorno físico particular que ofrece esta ciudad.

\section{Materiales y métodos}

\subsection{Aplicación de la Guía de Manejo Ambiental para el desarrollo de proyectos de infraestructura urbana en Bogotá D.C.}

Para contar con una estimación del impacto que los tratamientos silviculturales tienen sobre la función de anidación y crianza de las aves presentes en el área urbana de Bogotá, se seleccionó una muestra de 8 obras de infraestructura, hidráulicas y de orden vial, como Transmilenio, sistema de transporte masivo basado en la operación de buses con alta capacidad de usuarios que circulan por carriles de uso exclusivo y estaciones fijas ubicadas en los carriles centrales de las avenidas, para facilitar el acceso rápido de pasajeros, ambas, de gran impacto sobre el arbolado urbano. El criterio indispensable para la selección de las obras radicó en la existencia del reporte de manejo de avifauna, mediante la aplicación de la Guía (figura 1 y tabla 1 ). Se consultaron 19 expedientes, en el archivo de la SDA, por cuanto las obras fueron contratadas y monitoreadas en forma separada y sectorizada. La información tanto de la avifauna como de los árboles intervenidos con tala o traslado, contenida en los cuadros diligenciados con los datos recolectados en campo, fue procesada en hojas de cálculo Excel (v.16.0) con el fin de posibilitar su manejo aritmético y estadístico. La localización y espacialización de los transectos intervenidos fue realizada en el programa cartográfico ArcGIS versión 10.4. territarias 40 


\subsection{Presencia de especies de aves en relación con la matriz urbana de Bogotá}

Paralelamente a la obtención de los resultados preliminares del seguimiento al manejo de la avifauna en relación con la gestión del arbolado urbano de la ciudad, la autoridad ambiental de Bogotá decidió profundizar en la observación del estado de la biodiversidad de las aves en relación con la EEP existente en la ciudad. El trabajo "Criterios técnicos de conectividad ecológica con énfasis en la conservación de la avifauna y la consolidación de procesos de restauración en la estructura ecológica principal del Distrito Capital", producto del contrato-convenio 046 de 2007 entre la SDA y la ABO, realizó la caracterización de patrones de distribución y diversidad de comunidades de aves, así como la identificación de oportunidades de conservación y restauración de elementos naturales que permitan su presencia, movilidad y persistencia en la ciudad.

Para ello, identificó y seleccionó en la matriz urbana elementos naturales importantes en la conectividad estructural y funcional del paisaje que hicieran parte $o$ complementaran aquellos identificados bajo la EEP. Luego, se caracterizó la composición y estructura de las comunidades de aves asociadas con los elementos de paisaje seleccionados, a la vez que se identificaron las variables que condicionan la presencia o ausencia de especies de aves relacionadas con el paisaje circundante y la composición territarias 40 y estructura de la vegetación local. Así mismo, se realizó el levantamiento y revisión de la cartografía e imágenes satelitales Quickbird del año 2007, provistas por la SDA, así como la información cartográfica asociada principalmente con las coberturas de la EEP, del sistema de áreas protegidas, de parques urbanos dentro y fuera de la EEP, del sistema de ríos, cañadas y canales que atraviesan la ciudad, así como la cobertura de localidades, curvas de nivel, manzanas, vías y otros equipamientos.

Se identificaron 4 áreas denominadas "ventanas", utilizando criterios asociados principalmente con su ubicación geográfica, organización administrativa, tendencias climáticas y que presentaran mayores opciones de conservación y conectividad. Con la interpretación cartográfica de dichas ventanas, se elaboraron los mosaicos asegurando su cubrimiento total (figura 1).

Figura 1. Áreas de estudio - ventanas y obras de infraestructura

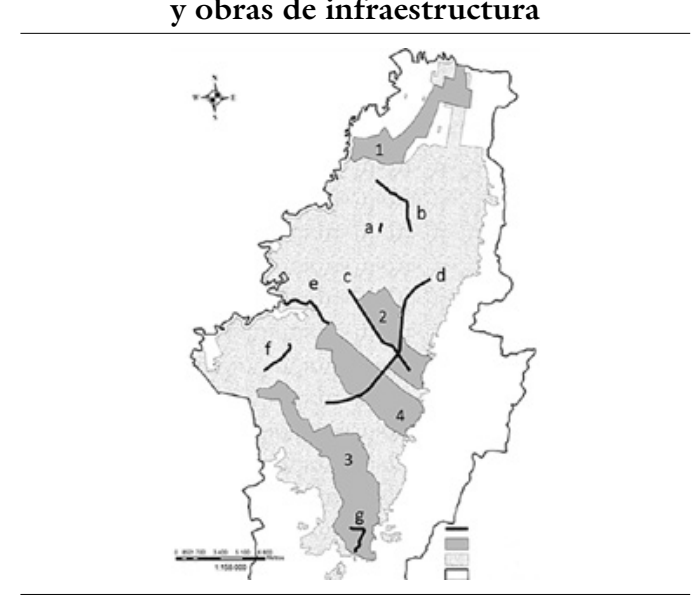


Posteriormente, se realizó la clasificación de paisajes, a partir de la agrupación de pixeles de niveles digitales espectralmente similares a una imagen. Así mismo, se realizaron los índices de vegetación para los mosaicos de cada ventana de trabajo, con el fin de realzar las áreas con mayor vigor vegetal (actividad fotosintética).

Se identificaron las áreas con mayor potencial de representar características relevantes para favorecer e incrementar la conectividad ecológica estructural y funcional de la ciudad, por medio de la localización de todos los parches de elementos considerados verdes presentes en cada ventana y se clasificaron como: corredores con vegetación, que incluye los corredores viales o férreos, cercas vivas, canales y ríos o quebradas; áreas verdes de uso público, que incluye parques y cementerios de gran tamaño, parques de tamaño intermedio, parques locales o de bolsillo y áreas mixtas; además de otras áreas verdes agrícolas, pecuarias y forestales; áreas verdes naturales y áreas altamente transformadas.

Las imágenes se digitalizaron y sirvieron para la consolidación de un mapa base para la interpretación del paisaje urbano, así como para la selección de áreas de muestreo, toma de información y análisis sobre la conectividad funcional para comunidades de aves presentes en el Distrito Capital.

En cada ventana se establecieron puntos de muestreo de aves con distancias de 200 metros entre sí, lo que arrojó un total de 940 puntos de muestreo (tabla 2). Se hicieron tres réplicas, por lo que el número total de puntos muestreados fue de 2820 .
Entre octubre de 2007 y abril de 2008 se realizó la observación ornitológica, entre las 6:00 y las 11:30 am. En un radio de $20 \mathrm{~m}$, en cada punto predefinido se colectó información de las actividades de las aves, tanto dentro como fuera del círculo de observación. Se aplicó el coeficiente de contingencia entre las categorías de abundancia de las especies de aves y las ventanas donde se avistaron; para establecer comparación de distribución entre ventanas se usó el índice de Jaccard (cualitativo); y para calcular la similaridad, el índice de Bray-Curtis (cuantitativo).

Respecto de la vegetación se realizaron 596 parcelas de muestreo. Con la información obtenida se efectuó un análisis de correspondencia con las abundancias de cada especie en las cuatro ventanas.

\section{Resultados}

\subsection{Manejo de Avifauna y del arbolado urbano de Bogotá, en construcción de infraestructura pública}

Las obras intervinieron en total 13925 árboles, 90,5\% talados y 9,5\% trasladados, solo se encontraron nidos en 1232 de ellos, correspondiente al $8,8 \%$. Se rescataron 1515 nidos, de los cuales 490 (32,3\%), cumplían su función, 312 contenían huevos y 178 contenían crías (tabla 1 ). Se rescataron 345 huevos y 231 juveniles de los cuales sobrevivieron 65, es decir el $28,1 \%$. No se rescataron 24 juveniles por estar a días de su madurez y autonomía, territarias 40 
Tabla 1 Manejo de la avifauna en relación con el número de árboles intervenidos por construcción de obras de infraestructura

\begin{tabular}{|c|c|c|c|c|c|c|c|c|c|c|c|c|c|}
\hline \multicolumn{2}{|c|}{ Obras de infraestructura } & 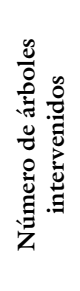 & 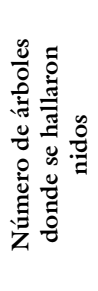 & 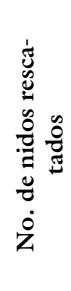 & 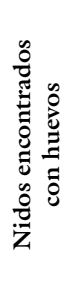 & 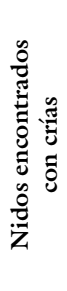 & 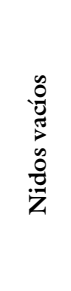 & 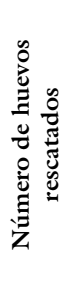 & 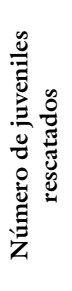 & 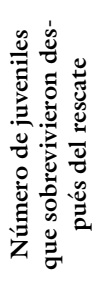 & 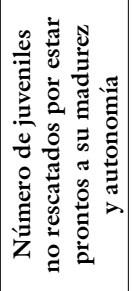 & 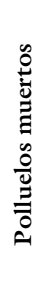 & 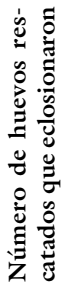 \\
\hline$a$ & $\begin{array}{l}\text { Construcción Av. Ciudad de Cali desde la Transversal } \\
91 \text { hasta el acceso barrio Lisboa. Construcción de dos } \\
\text { pontones vehiculares sobre el canal Salitre y el Hume- } \\
\text { dal Juan Amarillo. }\end{array}$ & 265 & 9 & 9 & 0 & 1 & 8 & 0 & 2 & 2 & 0 & 0 & 0 \\
\hline $\mathrm{b}$ & Transmilenio Av. Suba desde la Calle 127 al portal Suba & 5319 & 171 & 213 & 8 & 6 & 199 & 11 & 10 & 0 & 12 & 0 & 0 \\
\hline c & $\begin{array}{l}\text { Transmilenio Calle } 26 \text { desde la Transversal } 96 \text { a la } \\
\text { Carrera } 19\end{array}$ & 3809 & 453 & 560 & 49 & 62 & 449 & 150 & 85 & NR & NR & 10 & NR \\
\hline $\mathrm{d}$ & $\begin{array}{l}\text { Transmileno Av. NQS desde la Calle } 92 \text { a la Escuela } \\
\text { Militar General Santander }\end{array}$ & 4008 & 560 & 691 & 251 & 105 & 335 & 177 & 134 & 63 & 8 & 82 & 4 \\
\hline $\mathrm{e}$ & $\begin{array}{l}\text { Canalización Río Fucha desde la Av. Boyacá hasta el } \\
\text { Pondaje }\end{array}$ & 318 & 25 & 25 & 2 & 0 & 23 & 3 & 0 & 0 & 0 & 0 & 0 \\
\hline $\mathrm{f}$ & $\begin{array}{l}\text { Andenes y Ciclorruta Av. Agoberto Mejía entre las } \\
\text { calles } 13 \text { y } 53 \text { sur }\end{array}$ & 11 & 4 & 4 & 1 & 0 & 3 & 3 & 0 & 0 & 0 & 0 & NR \\
\hline $\mathrm{g}$ & Ampliación autopista al Llano desde $\mathrm{Kl}+575$ al $\mathrm{K} 5+324$ & 149 & 8 & 11 & 1 & 4 & 6 & 1 & 0 & 0 & 4 & 1 & NR \\
\hline $\mathrm{h}$ & $\begin{array}{l}\text { Vías para rutas alimentadoras Transmilenio, zona } \\
4 \text {-grupo } 1 \text { e Intervenciones integrales malla vial fase } \\
1 \text { grupo } 2 \text {-Suba; Grupo } 2 \text { Usme, Mártires, Santafé y } \\
\text { Rafael Uribe; Grupo } 3 \text { Kennedy; Grupo } 3 \text { fase } 2 \text { Tun- } \\
\text { juelito y Ciudad Bolívar }\end{array}$ & 46 & 2 & 2 & 0 & 0 & 2 & 0 & 0 & 0 & 0 & 0 & 0 \\
\hline & Total & 13925 & 1232 & 1515 & 312 & 178 & 1025 & 345 & 231 & 65 & 24 & 93 & 4 \\
\hline
\end{tabular}

NR: No reportado

Fuente: elaboración propia. Expedientes Secretaría Distrital de Ambiente.

territarias 40 90 tampoco 3 nidos por dificultad en la aproximación de la grúa y no se encontraron 13 nidos debido a la realización de podas previas al rescate.

Los árboles intervenidos en esa muestra correspondieron a 112 especies, cuya representación fue en su orden: ciprés $(\mathrm{Cu}$ pressus lusitánica) 1346, roble (Quercus humboldtii) 1334, urapán (Fraxinus chinensis) 1304, acacia japonesa (Acacia melanoxylum) 918, laurel huesito (Pittosphorun undulatum) 688, sauco (Sambucus peruviana) 677, caucho sabanero (Ficus andicola) 565, palma yuca (Yucca elephantipes) 546, cerezo (Prunus carica) 480, acacia negra (Acacia decurrens) 466, eucalipto 
común (Eucalyptus globulus) 432, holly liso (Pyracantha coccinea) 419, jazmín de la china (Ligustrum sp) 378, falso pimiento (Shinus molle) 376, guayacán de manizales (Lafoensia speciosa) 285, palma fénix (Phoenix canariensis) 181 y 3537 individuos de otras 96 especies.

La predilección de la avifauna denota que un poco más de la mitad de los anidamientos, el $52,7 \%$, se presentaron en solo cinco especies forestales, de las 50 en donde se hallaron nidos, estas son: urapán (F. chinensis) con $13,8 \%$, pino candelabro (P. radiata) con el $12 \%$, caucho sabanero (F. andicola) con el 10,7\%, eucalipto común (E. globulus) con $8,7 \%$ y el ciprés (C. lusitánica) con 7,5\%.

La presencia de nidos por especie forestal intervenida muestra que de los 127 individuos talados de la especie pino candelabro ( $P$. radiata), 99 tenían nido, es decir que hubo anidación en el $78 \%$ de los árboles de esta especie. A continuación, se muestra el porcentaje de anidación: sauce (Salix humboldtiana) 31,8\%, caucho sabanero (F. andicola) 30,1\%, caucho Tequendama (Ficus tequendamae) 23,1\%, acacia gris (A. melanoxylum) 21,9\%, eucalipto común (E. globulus) 21,8\%, caucho benjamín (F. benjamina) 18,7\%, pino monterrey (Pinus patula) 14,7\%, caucho común (Ficus elastica) 12,7\%, urapán (F. chinensis) 12,4\%, palma yuca ( $\Upsilon$. elephantipes) $9,2 \%$, chicalá (Tecoma stans) $8,1 \%$, sauco (S. peruviana) 7,6\%, acacia japonesa ( $A$. melanoxylum) 6\%, ciprés (C. Iusitanica) $5,4 \%$, jazmín del cabo (P. undulatum) $4,3 \%$, y falso pimiento (S. molle) $2,1 \%$.
En los reportes de avifauna que reposan en los expedientes de obras no se identificaron las especies de aves que hicieron uso del 47,2\% de los nidos rescatados. Además, se confirmó la actividad reproductiva sobre el arbolado urbano, de nueve especies de aves, siendo la torcaza (Zenaida auriculata), la especie que de lejos es la más común con una presencia del 37,6\% de los anidamientos, seguido por el copetón (Zonotrichia capensis) con $5,4 \%$, la mirla (Turdus fuscater) con $4 \%$, la chisga (Carduelis spinescens) con 1,7\%, los colibríes con 1,3\% y el sirirí (Tyrannus melancholicus), la paloma doméstica (Columba livia), el chamón (Molothrus bonariensis) y el canario bogotano (Sicalis flaveola), cada uno con $0,7 \%$.

\subsection{Relación de aves y vegetación, en asocio con la interpretación del paisaje urbano de Bogotá}

La caracterización florística y estructural de las ventanas estudiadas registró la presencia de 112 familias y 258 especies, con un total de 16237 individuos en los tres estratos, arbóreo (41,2\%), arbustivo (51,9\%) y herbáceo $(6,1 \%)$. Las especies que en conjunto representan más del $40 \%$ de registros en orden de abundancia fueron el eucalipto común (E.globulus), urapán ( $F$. chinensis), acacia gris (Acacia decurrens), sauco (S. peruviana), acacia japonesa ( $A$. melanoxylum), jazmín del cabo (P. undulatum) y el ciprés (C. Iusitanica). Las curvas de acumulación determinaron que la correspondencia de las abundancias de territarios 40 
cada especie para cada una de las cuatro ventanas tuvo un intervalo de confianza del $95 \%$.

La altura promedio del componente arbóreo fue de $5,86 \mathrm{~m}$, debido a que la mayoría de individuos registrados se encuentran en clases de altura menores a $10 \mathrm{~m}$. Asimismo, el diámetro a la altura del pecho (DAP) promedio fue de $0,27 \mathrm{~cm}$.

La composición y estructura de la vegetación de los diferentes sitios de muestreo presenta una predominancia de pocas especies que, al igual que en las aves, dominan la comunidad en términos de abundancia. Es el caso de especies, principalmente introducidas como el eucalipto (Eucalyptus sp), urapán ( $F$. chinensis) y sauco ( $S$. peruviana). En la ventana 1 predominan el sauco (S. peruviana) y el sauce (Salix humboldtina), en la ventana 3, el urapán (F. chinensis) y la acacia japonesa (A. melanoxylum) y las ventanas 2 y 4 son dominadas por el eucalipto (Eucalyptussp).
$\mathrm{Al}$ interior de las ventanas se registraron 39 familias de aves que agrupan a 153 especies, de las cuales $121(79 \%)$ son residentes permanentes y 32 (21\%) son migratorias. En total, se observaron 46149 individuos, registrados en los 940 puntos de muestreo $(3$ repeticiones $\mathrm{c} / \mathrm{u}$ ) durante las aproximadamente 470 horas de observación (10 min/punto).

Las familias representadas con mayor número de especies corresponden con las de los atrapamoscas o Tyrannidae con 24 $(15,7 \%)$, seguida de las tángaras o Thraupidae con $15(9,8 \%)$, las reinitas o Parulidae con $11(7,2 \%)$ y los semilleros o Emberizidae con $10(6,5 \%)$.

Seis especies reunieron más del $70 \%$ de los individuos registrados en todo el muestreo, la torcaza (Z. auriculata) con el 20,1\% (9204 individuos), el copetón ( $Z$. capensis) con el 16,1\% (7438 individuos), la golondrina vientrigris (Orochelidon murina) con el 10,6\% (4910 individuos),

Tabla 2. Áreas de estudio sobre presencia de aves en el arbolado urbano de Bogotá

\section{territarias 40}

\begin{tabular}{|c|c|c|c|c|c|c|c|c|c|}
\hline \multirow{2}{*}{ Ventanas } & \multirow{2}{*}{$\begin{array}{c}\text { Área } \\
\text { (Has) }\end{array}$} & \multicolumn{2}{|c|}{$\begin{array}{l}\text { Puntos de } \\
\text { Muestreo }\end{array}$} & \multicolumn{3}{|c|}{ Aves } & \multicolumn{3}{|c|}{ Vegetación } \\
\hline & & Aves & Veg & $\mathrm{Sp}$ & Familias & Individuos & $\mathrm{Sp}$ & Familias & Individuos \\
\hline $\begin{array}{l}\text { Cerros de Torca y la Conejera- } \\
\text { Humedal la Conejera }\end{array}$ & 2654,03 & 317 & 129 & 111 & 31 & 16219 & 155 & 74 & 2919 \\
\hline $\begin{array}{l}\text { Río Arzobispo-Parque Metropolitano } \\
\text { Simón Bolívar }\end{array}$ & 1767,35 & 157 & 124 & 81 & 29 & 7133 & 184 & 91 & 3770 \\
\hline $\begin{array}{l}\text { Parque Entrenubes-Parque Timiza y } \\
\text { Cementerio El Apogeo }\end{array}$ & 4695,92 & 329 & 275 & 115 & 33 & 14099 & 215 & 88 & 7105 \\
\hline Río Fucha-Fábrica Bavaria & 3688,92 & 137 & 68 & 56 & 21 & 8698 & 122 & 63 & 2443 \\
\hline & 12791,18 & 940 & 596 & 153 & 39 & 46149 & 258 & 112 & 16237 \\
\hline
\end{tabular}

Fuente: elaboración propia con base en el Informe Final Convenio 046 de 2007 entre SDA y ABO. 
la mirla (T. fuscater) con el 9,1\% (4200 individuos), la paloma de castilla (C. $\mathrm{li}$ via) con el 7,7\% (3567 individuos) y el colibrí común (Colibri coruscans) con el 6,9\% (3169 individuos). De otro lado, 33 especies $(22 \%)$ registraron solamente un individuo.

En cuanto a distribución espacial, más del $90 \%$ de las especies concurrió puntualmente en menos del $20 \%$ de los puntos muestreados presentándose como especies raras y con pocos individuos registrados. Las torcazas, copetones, golondrinas y mirlas son las únicas especies que muestran distribuciones generales con presencia en toda la ciudad. La distribución de las aves, en los corredores de vegetación de áreas urbanas, se presenta en este escrito mediante la siguiente clasificación: áreas urbanas, canales y corredores viales o férreos, parques locales o de bolsillo, parques de tamaño intermedio, y parques y cementerios de gran tamaño. Tanto la abundancia como la distribución de la avifauna bogotana se compendian en la tabla 3 .

El mayor número de especies se registró en los bosques y matorrales (87), seguido por los potreros y cultivos (85), seguido por las zonas de ríos y quebradas (79), después por las áreas mixtas (76),

Tabla 3. Abundancia y distribución de las aves en la zona urbana de Bogotá

\begin{tabular}{|c|c|c|c|c|c|c|c|c|c|c|c|c|c|}
\hline Nombre Común & Nombre Científico & \multicolumn{2}{|c|}{$\begin{array}{l}\text { Áreas } \\
\text { Urbanas }\end{array}$} & \multicolumn{2}{|c|}{ Canales } & \multicolumn{2}{|c|}{$\begin{array}{c}\text { Corredores } \\
\text { viales o } \\
\text { férreos }\end{array}$} & \multicolumn{2}{|c|}{$\begin{array}{c}\text { Parques } \\
\text { locales } \\
\text { o de } \\
\text { bolsillo }\end{array}$} & \multicolumn{2}{|c|}{$\begin{array}{c}\text { Parques } \\
\text { de tamaño } \\
\text { intermedio }\end{array}$} & \multicolumn{2}{|c|}{$\begin{array}{c}\text { Parques y } \\
\text { cementerios } \\
\text { de gran } \\
\text { tamaño }\end{array}$} \\
\hline Torcaza común & Zenaida auriculata & $\mathrm{PC}$ & $\mathrm{G}$ & $\mathrm{C}$ & G & $\mathrm{F}$ & G & $\mathrm{C}$ & G & $\mathrm{C}$ & G & $\mathrm{F}$ & G \\
\hline Golondrina ahumada & Orochelidon murina & $\mathrm{R}$ & $\mathrm{Pa}$ & $\mathrm{C}$ & M & $\mathrm{C}$ & Ex & $\mathrm{PC}$ & M & $\mathrm{PC}$ & $\mathrm{Pa}$ & $\mathrm{C}$ & M \\
\hline Mirla patinaranja & Turdus fuscater & $\mathrm{E}$ & $\mathrm{G}$ & $\mathrm{C}$ & G & $\mathrm{C}$ & G & $\mathrm{PC}$ & G & $\mathrm{C}$ & Ex & $\mathrm{C}$ & $\mathrm{G}$ \\
\hline Paloma doméstica & Columba livia & $\mathrm{R}$ & $\mathrm{Pa}$ & $\mathrm{C}$ & Ex & $\mathrm{C}$ & $\mathrm{Pa}$ & $\mathrm{C}$ & Ex & $\mathrm{C}$ & M & $\mathrm{R}$ & $\mathrm{Pu}$ \\
\hline Cucarachero común & Troglodytes aedon & $\mathrm{R}$ & $\mathrm{Pa}$ & $\mathrm{R}$ & $\mathrm{Pu}$ & PC & $\mathrm{Pa}$ & $\mathrm{R}$ & $\mathrm{Pu}$ & $\mathrm{E}$ & $\mathrm{Pu}$ & $\mathrm{C}$ & Ex \\
\hline Sirirí común & Tyrannus melancholicus & $\mathrm{R}$ & $\mathrm{Pa}$ & PC & Ex & PC & $\mathrm{Pa}$ & $\mathrm{E}$ & $\mathrm{Pa}$ & $\mathrm{PC}$ & M & $\mathrm{PC}$ & $\mathrm{Pa}$ \\
\hline Gallinazo común, Chulo & Coragyps atratus & $\mathrm{R}$ & $\mathrm{Pa}$ & PC & $\mathrm{Pa}$ & $\mathrm{PC}$ & $\mathrm{Pa}$ & $\mathrm{R}$ & $\mathrm{Pu}$ & $\mathrm{E}$ & $\mathrm{Pu}$ & $\mathrm{E}$ & $\mathrm{Pu}$ \\
\hline Monjita, Jilguero colombiano & Carduelis psaltria & $\mathrm{R}$ & $\mathrm{Pu}$ & $\mathrm{E}$ & $\mathrm{Pu}$ & $\mathrm{PC}$ & $\mathrm{Pa}$ & $\mathrm{R}$ & $\mathrm{Pu}$ & $\mathrm{E}$ & $\mathrm{Pu}$ & PC & $\mathrm{Pa}$ \\
\hline Atrapamoscas pechirrojo, Titiribí & Pyrocephalus rubinus & $\mathrm{R}$ & $\mathrm{Pu}$ & $\mathrm{R}$ & $\mathrm{Pu}$ & $\mathrm{R}$ & $\mathrm{Pu}$ & $\mathrm{R}$ & $\mathrm{Pu}$ & $\mathrm{E}$ & $\mathrm{Pu}$ & $\mathrm{E}$ & $\mathrm{Pu}$ \\
\hline Carbonero, picaflor negro & Diglossa humeralis & $\mathrm{R}$ & $\mathrm{Pa}$ & $\mathrm{R}$ & $\mathrm{Pu}$ & $\mathrm{E}$ & $\mathrm{Pa}$ & $\mathrm{R}$ & $\mathrm{Pu}$ & $\mathrm{R}$ & $\mathrm{Pu}$ & $\mathrm{E}$ & $\mathrm{Pu}$ \\
\hline
\end{tabular}




\begin{tabular}{|c|c|c|c|c|c|c|c|c|c|c|c|c|c|}
\hline \multirow[t]{2}{*}{ Nombre Común } & \multirow[t]{2}{*}{ Nombre Científico } & \multicolumn{2}{|c|}{$\begin{array}{c}\text { Áreas } \\
\text { Urbanas }\end{array}$} & \multicolumn{2}{|c|}{ Canales } & \multicolumn{2}{|c|}{$\begin{array}{c}\text { Corredores } \\
\text { viales o } \\
\text { férreos }\end{array}$} & \multicolumn{2}{|c|}{$\begin{array}{l}\text { Parques } \\
\text { locales } \\
\text { o de } \\
\text { bolsillo }\end{array}$} & \multicolumn{2}{|c|}{$\begin{array}{c}\text { Parques } \\
\text { de tamaño } \\
\text { intermedio }\end{array}$} & \multicolumn{2}{|c|}{$\begin{array}{c}\text { Parques y } \\
\text { cementerios } \\
\text { de gran } \\
\text { tamaño }\end{array}$} \\
\hline & & A & D & A & D & $\mathbf{A}$ & D & A & D & A & D & A & $\mathrm{D}$ \\
\hline Chirlobirlo & Sturnella magna & $\mathrm{R}$ & $\mathrm{Pa}$ & $\mathrm{R}$ & $\mathrm{Pu}$ & $\mathrm{E}$ & $\mathrm{Pu}$ & $\mathrm{R}$ & $\mathrm{Pu}$ & $\mathrm{R}$ & $\mathrm{Pu}$ & $\mathrm{E}$ & $\mathrm{Pu}$ \\
\hline Garcita bueyera & Bubulcus ibis & & & $\mathrm{E}$ & $\mathrm{Pu}$ & $\mathrm{PC}$ & $\mathrm{Pa}$ & $\mathrm{R}$ & $\mathrm{Pu}$ & $\mathrm{R}$ & $\mathrm{Pu}$ & $\mathrm{PC}$ & $\mathrm{Pa}$ \\
\hline $\begin{array}{l}\text { Chisga, Luganito andino, turpial } \\
\text { andino }\end{array}$ & Carduelis spinescens & & & $\mathrm{E}$ & $\mathrm{Pu}$ & $\mathrm{PC}$ & $\mathrm{Pa}$ & $\mathrm{R}$ & $\mathrm{Pu}$ & $\mathrm{E}$ & $\mathrm{Pu}$ & PC & $\mathrm{Pa}$ \\
\hline Picaflor pechicanelo & Diglossa sittoides & $\mathrm{R}$ & $\mathrm{Pu}$ & & & $\mathrm{R}$ & $\mathrm{Pu}$ & $\mathrm{R}$ & $\mathrm{Pu}$ & $\mathrm{R}$ & $\mathrm{Pu}$ & $\mathrm{E}$ & $\mathrm{Pu}$ \\
\hline Toche & Icterus chrysater & $\mathrm{R}$ & $\mathrm{Pu}$ & $\mathrm{R}$ & $\mathrm{Pu}$ & $\mathrm{R}$ & $\mathrm{Pu}$ & & & $\mathrm{R}$ & $\mathrm{Pu}$ & $\mathrm{E}$ & $\mathrm{Pu}$ \\
\hline Jilguero dorado & Sicalis flaveola & $\mathrm{R}$ & $\mathrm{Pu}$ & $\mathrm{R}$ & $\mathrm{Pu}$ & $\mathrm{E}$ & $\mathrm{Pu}$ & & & $\mathrm{R}$ & $\mathrm{Pu}$ & $\mathrm{R}$ & $\mathrm{Pu}$ \\
\hline Gavilán maromero & Elanus leucurus & & & $\mathrm{R}$ & $\mathrm{Pu}$ & $\mathrm{R}$ & $\mathrm{Pu}$ & $\mathrm{R}$ & $\mathrm{Pu}$ & $\mathrm{R}$ & $\mathrm{Pu}$ & $\mathrm{R}$ & $\mathrm{Pu}$ \\
\hline Tángara roja & Piranga rubra & & & $\mathrm{R}$ & $\mathrm{Pu}$ & $\mathrm{R}$ & $\mathrm{Pu}$ & $\mathrm{R}$ & $\mathrm{Pu}$ & $\mathrm{R}$ & $\mathrm{Pu}$ & $\mathrm{R}$ & $\mathrm{Pu}$ \\
\hline Azulejo común & Thraupis episcopus & & & $\mathrm{R}$ & $\mathrm{Pu}$ & $\mathrm{R}$ & $\mathrm{Pu}$ & $\mathrm{R}$ & $\mathrm{Pu}$ & $\mathrm{R}$ & $\mathrm{Pu}$ & $\mathrm{R}$ & $\mathrm{Pu}$ \\
\hline Reinita verderona & Vermivora peregrina & & & $\mathrm{R}$ & $\mathrm{Pu}$ & $\mathrm{R}$ & $\mathrm{Pu}$ & $\mathrm{R}$ & $\mathrm{Pu}$ & $\mathrm{R}$ & $\mathrm{Pu}$ & $\mathrm{R}$ & $\mathrm{Pu}$ \\
\hline Elenia & Elaenia frantzii & & & $\mathrm{R}$ & $\mathrm{Pu}$ & $\mathrm{E}$ & $\mathrm{Pu}$ & $\mathrm{R}$ & $\mathrm{Pu}$ & $\mathrm{R}$ & $\mathrm{Pu}$ & $\mathrm{E}$ & $\mathrm{Pu}$ \\
\hline Azulejo palmero & Thraupis palmarum & & & $\mathrm{E}$ & $\mathrm{Pu}$ & $\mathrm{R}$ & $\mathrm{Pu}$ & $\mathrm{R}$ & $\mathrm{Pu}$ & $\mathrm{E}$ & $\mathrm{Pu}$ & $\mathrm{R}$ & $\mathrm{Pu}$ \\
\hline $\begin{array}{l}\text { Piquito de oro, Semillero } \\
\text { coliblanco }\end{array}$ & Catamenia analis & $\mathrm{R}$ & $\mathrm{Pu}$ & $\mathrm{R}$ & $\mathrm{Pu}$ & $\mathrm{R}$ & $\mathrm{Pu}$ & $\mathrm{R}$ & $\mathrm{Pu}$ & & & & \\
\hline Cometa coliverde & Lesbia nuna & $\mathrm{R}$ & $\mathrm{Pu}$ & & & $\mathrm{R}$ & $\mathrm{Pu}$ & & & $\mathrm{R}$ & $\mathrm{Pu}$ & $\mathrm{E}$ & $\mathrm{Pu}$ \\
\hline Gavilán aliancho & Buteo platypterus & $\mathrm{R}$ & $\mathrm{Pu}$ & & & & & $\mathrm{R}$ & $\mathrm{Pu}$ & $\mathrm{R}$ & $\mathrm{Pu}$ & $\mathrm{R}$ & $\mathrm{Pu}$ \\
\hline Reinita gorginaranja & Dendroica fusca & & & $\mathrm{R}$ & $\mathrm{Pu}$ & $\mathrm{R}$ & $\mathrm{Pu}$ & & & $\mathrm{R}$ & $\mathrm{Pu}$ & $\mathrm{R}$ & $\mathrm{Pu}$ \\
\hline Tiranuelo gorgiblanco & Mecocerculus leucophrys & & & $\mathrm{R}$ & $\mathrm{Pu}$ & $\mathrm{R}$ & $\mathrm{Pu}$ & & & $\mathrm{R}$ & $\mathrm{Pu}$ & $\mathrm{E}$ & $\mathrm{Pu}$ \\
\hline Elaenia copetona & Elaenia flavogaster & & & $\mathrm{R}$ & $\mathrm{Pu}$ & & & $\mathrm{R}$ & $\mathrm{Pu}$ & $\mathrm{R}$ & $\mathrm{Pu}$ & $\mathrm{R}$ & $\mathrm{Pu}$ \\
\hline Mirla blanca, sinsonte común & Mimus gilvus & & & $\mathrm{R}$ & $\mathrm{Pu}$ & & & $\mathrm{R}$ & $\mathrm{Pu}$ & $\mathrm{R}$ & $\mathrm{Pu}$ & $\mathrm{R}$ & $\mathrm{Pu}$ \\
\hline Reinita dorada & Dendroica petechia & & & & & $\mathrm{R}$ & $\mathrm{Pu}$ & $\mathrm{R}$ & $\mathrm{Pu}$ & $\mathrm{R}$ & $\mathrm{Pu}$ & $\mathrm{R}$ & $\mathrm{Pu}$ \\
\hline Torcaza collareja & Patagioenas fasciata & $\mathrm{R}$ & $\mathrm{Pu}$ & & & $\mathrm{E}$ & $\mathrm{Pu}$ & $\mathrm{E}$ & $\mathrm{Pu}$ & & & & \\
\hline Oriol amarillo, Turpial amarillo & Icterus nigrogularis & $\mathrm{R}$ & $\mathrm{Pu}$ & & & & & & & $\mathrm{R}$ & $\mathrm{Pu}$ & $\mathrm{R}$ & $\mathrm{Pu}$ \\
\hline Cometa colinegro & Lesbia victoriae & $\mathrm{R}$ & $\mathrm{Pu}$ & & & & & $\mathrm{R}$ & $\mathrm{Pu}$ & & & $\mathrm{R}$ & $\mathrm{Pu}$ \\
\hline Monjita cabeciamarilla & Chrysomus icterocephalus & & & $\mathrm{R}$ & $\mathrm{Pu}$ & $\mathrm{E}$ & $\mathrm{Pu}$ & & & $\mathrm{R}$ & $\mathrm{Pu}$ & & \\
\hline $\begin{array}{l}\text { Sangre toro, cardenal, toche pico } \\
\text { de plata }\end{array}$ & Ramphocelus dimidiatus & & & $\mathrm{R}$ & $\mathrm{Pu}$ & & & & & $\mathrm{R}$ & $\mathrm{Pu}$ & $\mathrm{R}$ & $\mathrm{Pu}$ \\
\hline Atrapamoscas guardapuentes & Sayornis nigricans & & & $\mathrm{R}$ & $\mathrm{Pu}$ & & & $\mathrm{R}$ & $\mathrm{Pu}$ & & & $\mathrm{R}$ & $\mathrm{Pu}$ \\
\hline Tingua, Polla gris & Gallinula chloropus & & & $\mathrm{R}$ & $\mathrm{Pu}$ & & & & & & & $\mathrm{R}$ & $\mathrm{Pu}$ \\
\hline
\end{tabular}




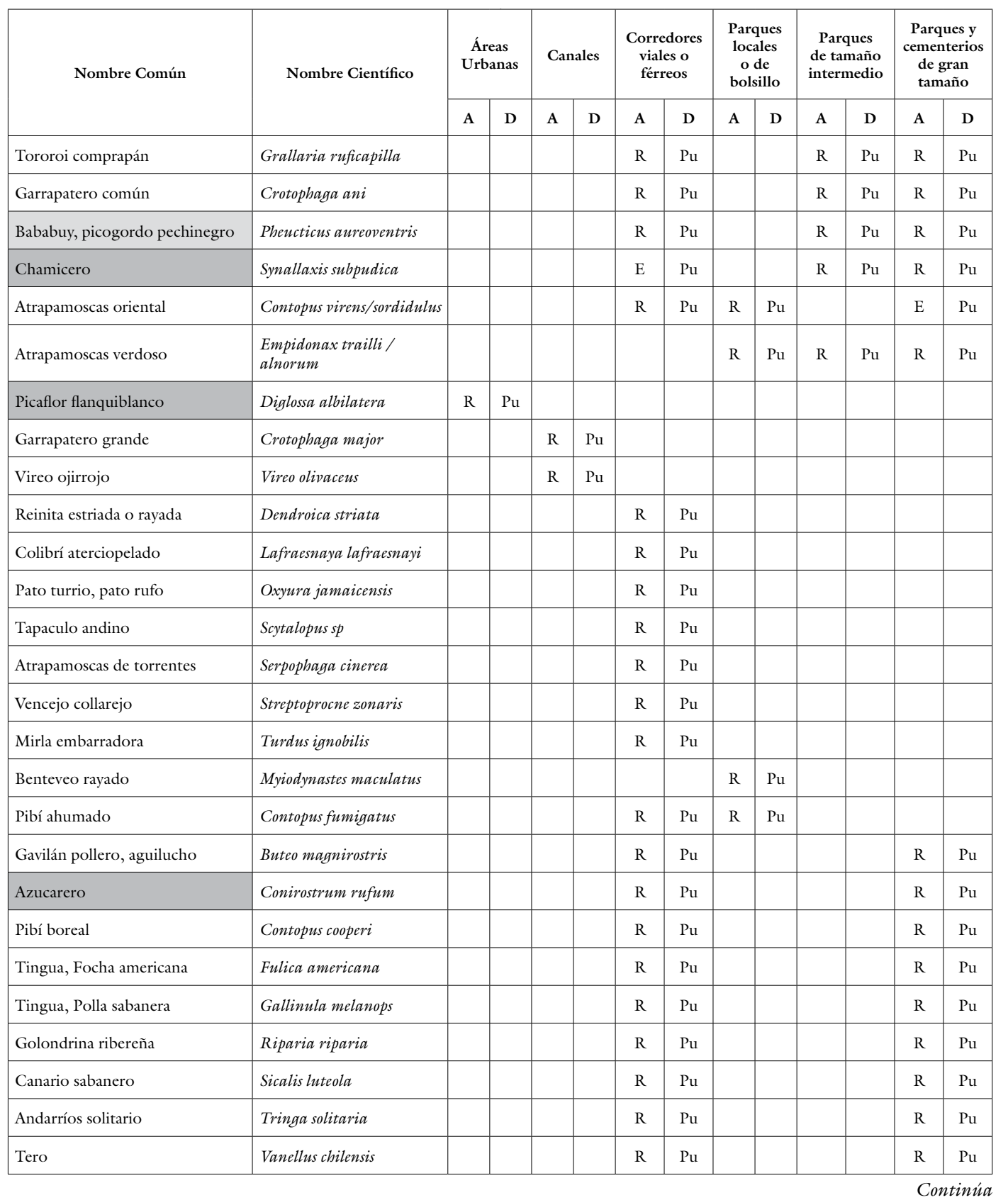

Manejo de la avifauna como parte de la gestión del arbolado urbano en Bogotá D.C. 


\begin{tabular}{|c|c|c|c|c|c|c|c|c|c|c|c|c|c|}
\hline \multirow[t]{2}{*}{ Nombre Común } & \multirow[t]{2}{*}{ Nombre Científico } & \multicolumn{2}{|c|}{$\begin{array}{l}\text { Áreas } \\
\text { Urbanas }\end{array}$} & \multicolumn{2}{|c|}{ Canales } & \multicolumn{2}{|c|}{$\begin{array}{l}\text { Corredores } \\
\text { viales o } \\
\text { férreos }\end{array}$} & \multicolumn{2}{|c|}{$\begin{array}{l}\text { Parques } \\
\text { locales } \\
\text { o de } \\
\text { bolsillo }\end{array}$} & \multicolumn{2}{|c|}{$\begin{array}{c}\text { Parques } \\
\text { de tamaño } \\
\text { intermedio }\end{array}$} & \multicolumn{2}{|c|}{$\begin{array}{c}\text { Parques y } \\
\text { cementerios } \\
\text { de gran } \\
\text { tamaño }\end{array}$} \\
\hline & & A & D & A & D & A & D & A & D & A & $\mathrm{D}$ & A & $\mathrm{D}$ \\
\hline $\begin{array}{l}\text { Atajacaminos ñañarca, gallina } \\
\text { ciega }\end{array}$ & Caprimulgus longirostris & & & & & & & $\mathrm{R}$ & $\mathrm{Pu}$ & & & $\mathrm{R}$ & $\mathrm{Pu}$ \\
\hline Garcita rayada & Butorides striata & & & & & & & & & $\mathrm{R}$ & $\mathrm{Pu}$ & $\mathrm{R}$ & $\mathrm{Pu}$ \\
\hline Garza patiamarilla & Egretta thula & & & & & & & & & $\mathrm{R}$ & $\mathrm{Pu}$ & $\mathrm{R}$ & $\mathrm{Pu}$ \\
\hline Halconcito colorado & Falco sparverius & & & & & & & & & $\mathrm{R}$ & $\mathrm{Pu}$ & $\mathrm{R}$ & $\mathrm{Pu}$ \\
\hline Turpial venezolano & Icterus icterus & & & & & & & & & $\mathrm{R}$ & $\mathrm{Pu}$ & $\mathrm{R}$ & $\mathrm{Pu}$ \\
\hline Saíra de antifaz & Pipraeidea melanonota & & & & & & & & & $\mathrm{R}$ & $\mathrm{Pu}$ & $\mathrm{R}$ & $\mathrm{Pu}$ \\
\hline Tángara escarlata & Piranga olivacea & & & & & & & & & $\mathrm{R}$ & $\mathrm{Pu}$ & $\mathrm{R}$ & $\mathrm{Pu}$ \\
\hline Zambullidor piquigrueso & Podilymbus podiceps & & & & & & & & & $\mathrm{R}$ & $\mathrm{Pu}$ & $\mathrm{R}$ & $\mathrm{Pu}$ \\
\hline Arañero cabecinegro & Basileuterus nigrocristatus & & & & & & & & & & & $\mathrm{R}$ & $\mathrm{Pu}$ \\
\hline Zumbador ventriblanco & Chaetocercus mulsant & & & & & & & & & & & $\mathrm{R}$ & $\mathrm{Pu}$ \\
\hline Picaflor de antifaz & Diglossa cyanea & & & & & & & & & & & $\mathrm{R}$ & $\mathrm{Pu}$ \\
\hline Esmerejón & Falco columbarius & & & & & & & & & & & $\mathrm{R}$ & $\mathrm{Pu}$ \\
\hline Golondrina común o tijereta & Hirundo rustica & & & & & & & & & & & $\mathrm{R}$ & $\mathrm{Pu}$ \\
\hline Carpintero habado & Melanerpes rubricapillus & & & & & & & & & & & $\mathrm{R}$ & $\mathrm{Pu}$ \\
\hline Martinete común, Guaco común & Nycticorax nycticorax & & & & & & & & & & & $\mathrm{R}$ & $\mathrm{Pu}$ \\
\hline Carpintero pardo & Picoides fumigatus & & & & & & & & & & & $\mathrm{R}$ & $\mathrm{Pu}$ \\
\hline Benteveo común & Pitangus sulphuratus & & & & & & & & & & & $\mathrm{R}$ & $\mathrm{Pu}$ \\
\hline Tingua azul & Porphyrio martinica & & & & & & & & & & & $\mathrm{R}$ & $\mathrm{Pu}$ \\
\hline Polluela sora & Porzana carolina & & & & & & & & & & & $\mathrm{R}$ & $\mathrm{Pu}$ \\
\hline Tijereta sabanera & Tyrannus savana & & & & & & & & & & & $\mathrm{R}$ & $\mathrm{Pu}$ \\
\hline Tirano viajero, sirirí migrante & Tyrannus tyrannus & & & & & & & & & & & $\mathrm{R}$ & $\mathrm{Pu}$ \\
\hline
\end{tabular}

A= Abundancia: A=Abundante, F=Frecuente, $C=$ Común

D= Distribución: $\mathrm{G}=$ General, Ex=Extensa, $M=$ Media,

$\mathrm{PC}=$ Poco Común, E= Escasa, $\mathrm{R}=$ Rara

$\mathrm{Pa}=\mathrm{Parche}, \mathrm{Pu}=$ Puntual

Chisga, Luganito andino, turpial Especie con posibilidad de colonización de la ciudad, aplicando el precepto de arborización urbana andino

Picaflor pechicanelo

Especie considerada como de alto valor e interés de conservación

Fuente: elaboración propia con base en el Informe Final Convenio de 2007 entre SDA y ABO.

\section{territarios 40}


los parques y cementerios de gran tamaño (75). El resto de los elementos presentó menos de 60 especies.

La relación de aves y vegetación, en asocio con la interpretación del paisaje urbano, evidencia que la ventana 4 tiene un valor en términos de parches y de áreas de tipo urbano significativamente superior ( $>70 \%$ ) en relación con las demás, que no superaron el $40 \%$. En la ventana 1, en su mayoría coincidente con el área de la reserva forestal Thomas Van der Hammen, la predominancia de una matriz constituida principalmente por potreros y cultivos, así como áreas consideradas como mixtas, presentan una influencia de las especies más abundantes, donde el copetón (Z.capensis) y la mirla (T. fuscater) predominan en relación con la torcaza (Z. auriculata) que es la especie más abundante en las otras tres ventanas. La ventana 2 tiene una presencia considerable de elementos asociados con corredores lineales, viales y férreos, así como un significativo número de parques intermedios y de bolsillo. Esto difiere de la ventana 3 , donde los bosques y matorrales nativos son predominantes, en extensión, aunque en número son reducidos.

Respecto de las categorías de abundancia, muchas de las especies de aves son clasificadas como raras o escasas en el contexto urbano y son muy pocas las que se encuentran de forma frecuente, común o poco común.

Las especies avistadas en todas las áreas de observación son consideradas como abundantes o comunes y de distribución general para la ciudad, en este grupo se en- cuentran: torcaza común (Z. auriculata), copetón o gorrión (Z. capensis), golondrina ahumada (O. murina), mirla patinaranja (T. fuscater), paloma doméstica (C. livia), colibrí chillón (C. coruscans), chamón común (M. bonariensis), cucarachero común (Troglodytes aedon), sirirí común (T. melancholicus), gallinazo común o chulo (Coragyps atratus), monjita o jilguero colombiano (Carduelis psaltria), atrapamoscas pechirrojo (Pyrocephalus rubinus), carbonero o picaflor negro (Diglossa bumeralis) y chirlobirlo (Sturnella magna).

Las especies consideradas como raras fueron registradas en una o pocas oportunidades, estas se encuentran en la periferia de la ciudad donde el proceso de degradación ha sido menos agresivo. Algunas son consideradas de alto valor o interés de conservación como: picaflor pechicanelo (Diglossa sittoides), toche (Icterus chrysater), tiranuelo gorgiblanco (Mecocerculus leucophrys), saíra de antifaz (Pipraeidea melanonota), picaflor flanquiblanco (Diglossa albilatera) y picaflor de antifaz (Diglossa cyanea); además, por su calidad endémica, el chamicero (Synallaxis subpudica), azucarero (Conirostrum rufum), colibrí (Eriocnemis cupreoventris), inca buchidorado (Coeligena bonapartei), inca ventrivioleta (Coeligena helianthea) y tapaculo andino (Scytalopus spp). Otras como el arañero cabecinegro (Basileuterus nigrocristatus), hemispingus cejiblanco (Hemispingus superciliaris), y el gorrión montés cabeciblanco (Atlapetes pallidinucha) se encuentran amenazadas a nivel global o nacional. territarias 40 
La riqueza de avifauna en relación con la interpretación del paisaje denota que las ventanas con menores intensidades de urbanización y con mayor presencia de bosques, matorrales y parches en áreas de uso público son aquellas donde la diversidad de la cobertura vegetal fue mayor. Ello es tangible en parques y cementerios de gran tamaño; el caso contrario se observa en canales y parques de bolsillo, donde las coberturas son de baja diversidad de especies.

En las áreas asociadas con espacios de uso público, parques y corredores ecológicos de ronda o canales, la riqueza de especies es baja dado a que la comunidad de aves tiende a conformarse de las especies ampliamente distribuidas en la ciudad. La cobertura vegetal en estos sitios presenta bajas posibilidades para la conectividad de la avifauna, por ello la conectividad funcional entre los elementos de la EEP de la ciudad debe empezar por conocer la estructura del paisaje urbano y las relaciones árboles-aves en términos de alimento, anidación y soporte, con el fin de elegir los árboles adecuados para ser plantados en áreas que realmente ofrezcan oportunidades de conservación y conectividad (tabla 4 ).

Tabla 4. Relación especies arbóreas con especies de aves, en el área urbana de Bogotá

\begin{tabular}{|c|c|c|c|c|c|c|c|c|c|c|c|c|c|c|c|c|c|}
\hline \multicolumn{2}{|c|}{ Especie forestal } & \multicolumn{2}{|c|}{ Especie de ave } & \multicolumn{6}{|c|}{ Relación ave/árbol } & \multicolumn{3}{|c|}{ Origen } & \multicolumn{5}{|c|}{ Habitat } \\
\hline N. Común & N. Científico & N. Común & N. Científico & 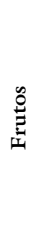 & 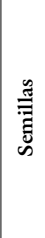 & $\frac{\mathscr{3}}{\frac{0}{x}}$ & 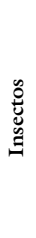 & 范 & 苞 & 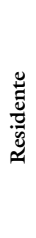 & 莺 & 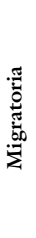 & 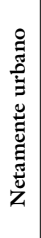 & 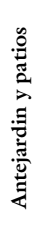 & 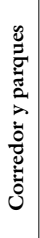 & 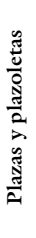 & 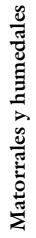 \\
\hline $\begin{array}{l}\text { Caucho } \\
\text { Sabanero, Gaque }\end{array}$ & $\begin{array}{l}\text { Ficus soatensis, Clusia } \\
\text { multiflora }\end{array}$ & Arrendajo & Cacicus cela & $\mathrm{X}$ & & & $\mathrm{X}$ & $\mathrm{X}$ & & & $\mathrm{X}$ & & & & $\mathrm{X}$ & & \\
\hline Cerezo & Prunus serotina & $\begin{array}{l}\text { Atrapamoscas } \\
\text { canelo, }\end{array}$ & $\begin{array}{l}\text { Pyrrhomyas } \\
\text { cinnamomeus }\end{array}$ & & & & $\mathrm{X}$ & $\mathrm{X}$ & & $\mathrm{X}$ & & & & & & & $\mathrm{X}$ \\
\hline $\begin{array}{l}\text { Pino romerón, } \\
\text { Roble }\end{array}$ & $\begin{array}{l}\text { Nageia rospigliossi, } \\
\text { Quercus humboldtii }\end{array}$ & $\begin{array}{l}\text { Mosquero } \\
\text { cardenal }\end{array}$ & $\begin{array}{l}\text { Pyrocephalus } \\
\text { rubinus }\end{array}$ & $\mathrm{X}$ & & & $\mathrm{X}$ & $\mathrm{X}$ & & & & $\mathrm{X}$ & & $\mathrm{X}$ & $\mathrm{X}$ & & \\
\hline $\begin{array}{l}\text { Abutilón, } \\
\text { Mangle de tierra } \\
\text { fría }\end{array}$ & $\begin{array}{l}\text { Abutilon insigne, } \\
\text { Escallonia pendula }\end{array}$ & Azucarero & $\begin{array}{l}\text { Conirostrum } \\
\text { rufum }\end{array}$ & & & $\mathrm{X}$ & & $\mathrm{X}$ & & & $\mathrm{X}$ & & & & $\mathrm{X}$ & & \\
\hline $\begin{array}{l}\text { Alcaparro, } \\
\text { Brevo, Caucho } \\
\text { Sabanero, } \\
\text { Cerezo, } \\
\text { Durazno, Feijoa, } \\
\text { Manzano }\end{array}$ & $\begin{array}{l}\text { Senna viarum, } \\
\text { Ficus carica, Ficus } \\
\text { soatensis, Prunus } \\
\text { serotina, Prunus } \\
\text { persica, Acca } \\
\text { sellowiana, Billia } \\
\text { colombiana }\end{array}$ & Azulejo & $\begin{array}{l}\text { Thraupis } \\
\text { episcopus }\end{array}$ & $\mathrm{X}$ & & & $\mathrm{X}$ & & & $\mathrm{X}$ & & & & $\mathrm{X}$ & $\mathrm{X}$ & & $\mathrm{X}$ \\
\hline
\end{tabular}

\section{territarias 40}




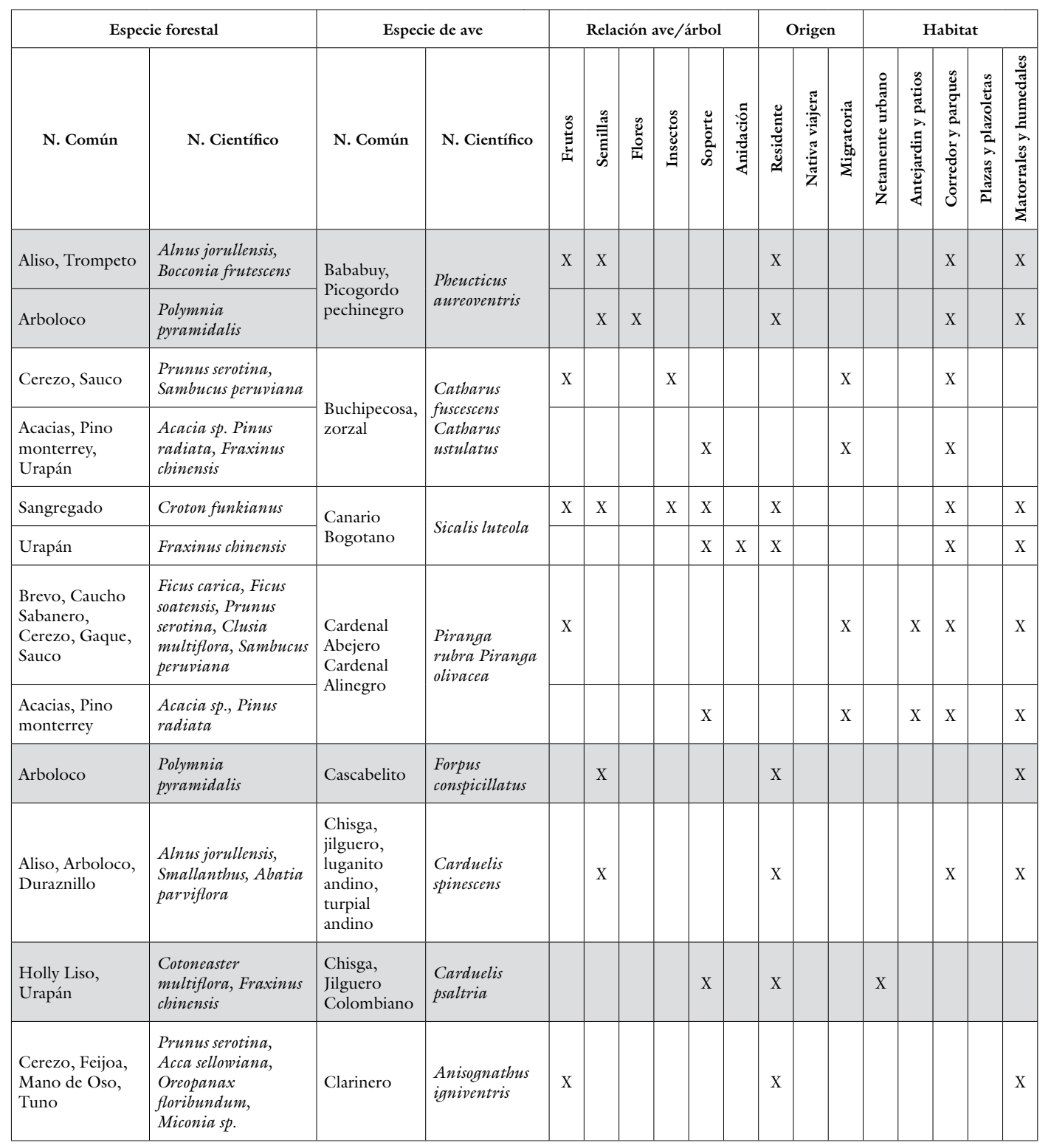

Continúa 


\begin{tabular}{|c|c|c|c|c|c|c|c|c|c|c|c|c|c|c|c|c|c|}
\hline \multicolumn{2}{|c|}{ Especie forestal } & \multicolumn{2}{|c|}{ Especie de ave } & \multicolumn{6}{|c|}{ Relación ave/árbol } & \multicolumn{3}{|c|}{ Origen } & \multicolumn{5}{|c|}{ Habitat } \\
\hline N. Común & N. Científico & N. Común & N. Científico & $\stackrel{\mathscr{B}}{*}$ & 节 & $\frac{\stackrel{e}{0}}{\overrightarrow{0}}$ & 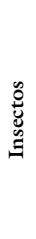 & 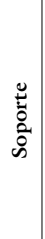 & 总 & 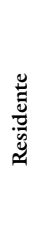 & 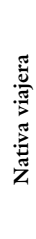 & 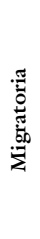 & 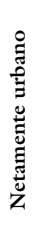 & 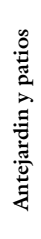 & 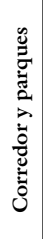 & 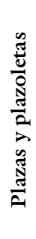 & 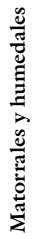 \\
\hline $\begin{array}{l}\text { Abutilón, } \\
\text { Alcaparro, } \\
\text { Borrachero, } \\
\text { Carbonero rojo, } \\
\text { chicalá, chocho, } \\
\text { duraznillo, } \\
\text { Eucalipto, } \\
\text { Gaque, } \\
\text { Guayacán de } \\
\text { Manizales, } \\
\text { Mangle, } \\
\text { Mermelada, } \\
\text { Sietecueros } \\
\text { Nazareno, } \\
\text { Zarcillejo }\end{array}$ & $\begin{array}{l}\text { Abutilon insigne, } \\
\text { Senna viarum, } \\
\text { Brugmansia } \\
\text { spp, Calliandra } \\
\text { carbonaria, Tecoma } \\
\text { stans, Erythrina } \\
\text { rubrinervia, } \\
\text { Abatia parviflora, } \\
\text { Eucalyptus sp., } \\
\text { Clusia multiflora, } \\
\text { Laphoensia speciosa, } \\
\text { Escallonia pendula, } \\
\text { Streptosolen } \\
\text { jamesonii, } \\
\text { Tibouchina } \\
\text { urvelleana, Fuchia } \\
\text { boliviana }\end{array}$ & Colibrí & $\begin{array}{l}\text { Colibris: } \\
\text { Géneros } \\
\text { Archilochus, } \\
\text { Calypte, } \\
\text { Chaetocercus, } \\
\text { Colibri, Lesbia, } \\
\text { Chaetocercus, } \\
\text { Ensifera, } \\
\text { Eugenes, } \\
\text { Ramphomicron, } \\
\text { Metallura, } \\
\text { Eriocnemis, } \\
\text { Trochilidae }\end{array}$ & & & $\mathrm{X}$ & & & & $\mathrm{X}$ & & & & $\mathrm{X}$ & $\mathrm{X}$ & & $\mathrm{X}$ \\
\hline $\begin{array}{l}\text { Aliso, Trompeto, } \\
\text { Gramíneas }\end{array}$ & $\begin{array}{l}\text { Alnus jorullensis, } \\
\text { Bocconia frutescens, } \\
\text { Chusquea sp. }\end{array}$ & Copetón & $\begin{array}{l}\text { Zonotrichia } \\
\text { capensis }\end{array}$ & & $\mathrm{X}$ & & & & & & & & $\mathrm{X}$ & $\mathrm{X}$ & $\mathrm{X}$ & $\mathrm{X}$ & \\
\hline $\begin{array}{l}\text { Cerezo, Mano } \\
\text { de Oso, Tuno }\end{array}$ & $\begin{array}{l}\text { Prunus serotina, } \\
\text { Oreopanax } \\
\text { floribundum. } \\
\text { Miconia sp. }\end{array}$ & Cotinga & $\begin{array}{l}\text { Ampelion } \\
\text { rubrocristatus }\end{array}$ & $\mathrm{X}$ & & & & & & $\mathrm{X}$ & & & & & & & $\mathrm{X}$ \\
\hline $\begin{array}{l}\text { Falso pimiento, } \\
\text { Feijoa, Mano } \\
\text { de Oso }\end{array}$ & $\begin{array}{l}\text { Schinus molle, } \\
\text { Acca sellowiana, } \\
\text { Oreopanax } \\
\text { floribundum }\end{array}$ & Elaenia & $\begin{array}{l}\text { Elaenia albi- } \\
\text { ceps, Elaenia } \\
\text { spectabilis, } \\
\text { Elaenia meso- } \\
\text { leuca, Elaenia } \\
\text { parvirostris, } \\
\text { Elaenia } \\
\text { Frantzii }\end{array}$ & $\mathrm{X}$ & & & & & & & $\mathrm{X}$ & & & & $\mathrm{X}$ & & \\
\hline $\begin{array}{l}\text { Roble } \\
\text { Australiano }\end{array}$ & Grevillea robusta & & $\begin{array}{l}\text { Elaenia } \\
\text { albiceps }\end{array}$ & & & $\mathrm{X}$ & & & & & $\mathrm{X}$ & & & & $\mathrm{X}$ & & \\
\hline Aliso & Alnus jorullensis & Espiguero & $\begin{array}{l}\text { Sporophila } \\
\text { luctuosa }\end{array}$ & $\mathrm{X}$ & $\mathrm{X}$ & & & & & $\mathrm{X}$ & & & & & $\mathrm{X}$ & & \\
\hline \multirow{2}{*}{ Feijoa } & \multirow{2}{*}{ Acca sellowiana } & Frutero azul & $\begin{array}{l}\text { Stephanophorus } \\
\text { diadematus }\end{array}$ & $\mathrm{X}$ & & & & & & & $\mathrm{X}$ & & & & $\mathrm{X}$ & & $\mathrm{X}$ \\
\hline & & Copetón & $\begin{array}{l}\text { Zonotrichia } \\
\text { capensis }\end{array}$ & $\mathrm{X}$ & & & & & & & & & $\mathrm{X}$ & $\mathrm{X}$ & $\mathrm{X}$ & $\mathrm{X}$ & \\
\hline Gaque & Clusia multiflora & Garrapatero & $\begin{array}{l}\text { Crotophanga } \\
\text { major }\end{array}$ & & & & & $\mathrm{X}$ & & & $\mathrm{X}$ & & & & $\mathrm{X}$ & & $\mathrm{X}$ \\
\hline
\end{tabular}




\begin{tabular}{|c|c|c|c|c|c|c|c|c|c|c|c|c|c|c|c|c|c|}
\hline \multicolumn{2}{|c|}{ Especie forestal } & \multicolumn{2}{|c|}{ Especie de ave } & \multicolumn{6}{|c|}{ Relación ave/árbol } & \multicolumn{3}{|c|}{ Origen } & \multicolumn{5}{|c|}{ Habitat } \\
\hline N. Común & N. Científico & N. Común & N. Científico & $\stackrel{0}{\stackrel{0}{2}}$ & 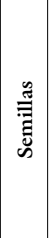 & 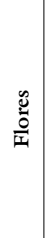 & $\stackrel{\mathscr{s}}{\mathscr{U}_{0}}$ & 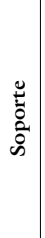 & 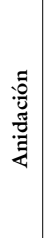 & 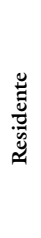 & 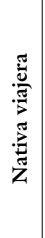 & 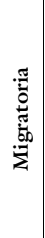 & 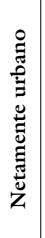 & 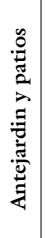 & 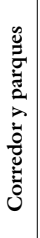 & 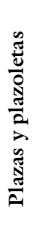 & 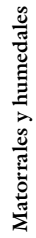 \\
\hline \multirow{6}{*}{$\begin{array}{l}\text { Ciprés, Eucalipto } \\
\text { común, Pino } \\
\text { Candelabro, } \\
\text { Urapán, Acacias, } \\
\text { Roble, Sauce }\end{array}$} & \multirow{6}{*}{$\begin{array}{l}\text { Cupressus lusitanica, } \\
\text { Eucalyptus globulus, } \\
\text { Pino radiata, } \\
\text { Fraxinus chinensis, } \\
\text { Quercus humboldtii, } \\
\text { Salix humboldtiana }\end{array}$} & $\begin{array}{l}\text { Gavilán de } \\
\text { alas anchas }\end{array}$ & $\begin{array}{l}\text { Buteo } \\
\text { platypterus }\end{array}$ & & & & & $\mathrm{X}$ & & & & $\mathrm{X}$ & & & $\mathrm{X}$ & & $\mathrm{X}$ \\
\hline & & $\begin{array}{l}\text { Gavilán } \\
\text { Grillero }\end{array}$ & $\begin{array}{l}\text { Buteo } \\
\text { magnirostris }\end{array}$ & & & & & $\mathrm{X}$ & & $\mathrm{X}$ & & & & & $\mathrm{X}$ & & $\mathrm{X}$ \\
\hline & & $\begin{array}{l}\text { Aguila de } \\
\text { Cabeza } \\
\text { Amarilla }\end{array}$ & $\begin{array}{l}\text { Milvago } \\
\text { chimachima }\end{array}$ & & & & & $\mathrm{X}$ & & $\mathrm{X}$ & & & & & $\mathrm{X}$ & & $\mathrm{X}$ \\
\hline & & $\begin{array}{l}\text { Bichofué } \\
\text { gritón }\end{array}$ & $\begin{array}{l}\text { Pitangus } \\
\text { sulphuratus }\end{array}$ & $\mathrm{X}$ & & & & $\mathrm{X}$ & & & $\mathrm{X}$ & & & & $\mathrm{X}$ & & \\
\hline & & Lechuza & Tyto alba & & & & & $\mathrm{X}$ & & $\mathrm{X}$ & & & & & $\mathrm{X}$ & & $\mathrm{X}$ \\
\hline & & $\begin{array}{l}\text { Reinita } \\
\text { gorginaranja }\end{array}$ & $\begin{array}{l}\text { Dendroica } \\
\text { fusca }\end{array}$ & & & & $\mathrm{X}$ & $\mathrm{X}$ & & & & $\mathrm{X}$ & & & $\mathrm{X}$ & & \\
\hline Trompeto & Bocconia frutescens & $\begin{array}{l}\text { Gorrión } \\
\text { cabeciblanco }\end{array}$ & $\begin{array}{l}\text { Atlapetes } \\
\text { pallidinucha }\end{array}$ & & $\mathrm{X}$ & & & & & $\mathrm{X}$ & & & & & & & $\mathrm{X}$ \\
\hline $\begin{array}{l}\text { Arrayán, } \\
\text { Corono, } \\
\text { Durazno, } \\
\text { Eugenia, Holly } \\
\text { espinoso, Laurel } \\
\text { huesito, Mano } \\
\text { de Oso, Palma } \\
\text { bayoneta, Palma } \\
\text { fénix, Sauco y } \\
\text { Trompeto }\end{array}$ & $\begin{array}{l}\text { Myrcianthes } \\
\text { leucoxyla, Xylosma } \\
\text { spiculiferum, } \\
\text { Prunus persica, } \\
\text { Eugenia myrtifolia, } \\
\text { Pyracantha } \\
\text { angustifolia, } \\
\text { Phittosporum } \\
\text { undulatum, } \\
\text { Oreopanax } \\
\text { floribundum, Yucca } \\
\text { elephantipes regel, } \\
\text { Phoenix canariensis, } \\
\text { Sambucus peruviana, } \\
\text { Bocconia frutescens. }\end{array}$ & Mirla & $\begin{array}{l}\text { Turdus } \\
\text { fuscater, } \\
\text { Turdus } \\
\text { ignobilis }\end{array}$ & $\mathrm{X}$ & & & & & & $\mathrm{X}$ & & & $\mathrm{X}$ & & $\mathrm{X}$ & $\mathrm{X}$ & \\
\hline $\begin{array}{l}\text { Roble, } \\
\text { Sangregado }\end{array}$ & $\begin{array}{l}\text { Quercus humboldtii, } \\
\text { Croton funkianus }\end{array}$ & Oriol & Icterus & & & & & $\mathrm{X}$ & & $\mathrm{X}$ & & & & & $\mathrm{X}$ & & \\
\hline Gaque & Clusia multiflora & Amnarino & arts & & & & $\mathrm{X}$ & $\mathrm{X}$ & & $\mathrm{X}$ & & & & & $\mathrm{X}$ & & \\
\hline $\begin{array}{l}\text { Brevo, Cerezo, } \\
\text { Durazno, Feijoa }\end{array}$ & $\begin{array}{l}\text { Ficus carica, Prunus } \\
\text { serotina, Prunus } \\
\text { persica, Acca } \\
\text { sellowiana }\end{array}$ & Oropéndola & $\begin{array}{l}\text { Gymnomystax } \\
\text { mexicanus }\end{array}$ & $\mathrm{X}$ & & & & & & & $\mathrm{X}$ & & & & $\mathrm{X}$ & & \\
\hline $\begin{array}{l}\text { Acacias, } \\
\text { Chusque }\end{array}$ & $\begin{array}{l}\text { Acacia sp., Chusquea } \\
\text { sp. }\end{array}$ & & & & & & & $\mathrm{X}$ & & & $\mathrm{X}$ & & & & $\mathrm{X}$ & & \\
\hline
\end{tabular}




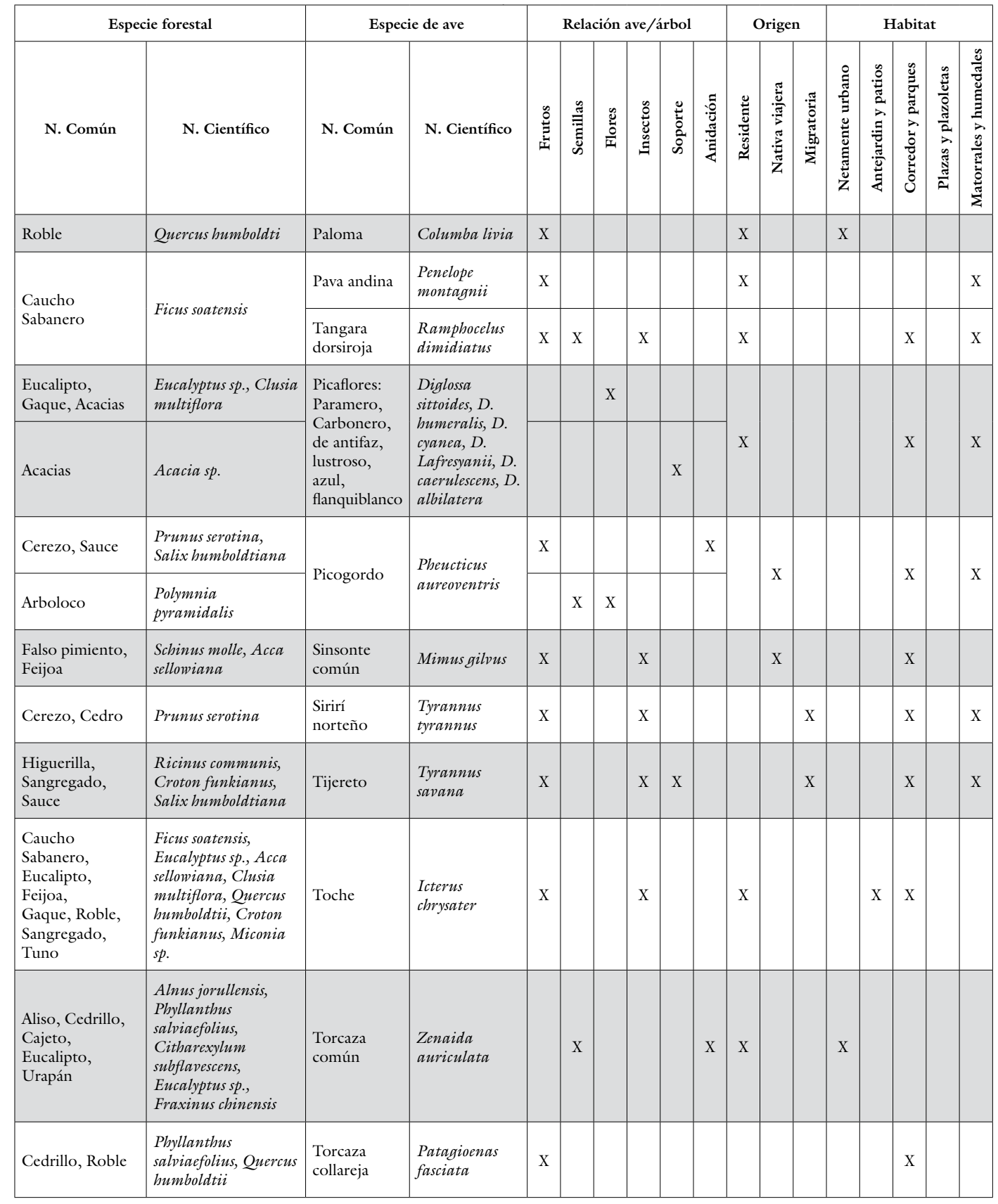




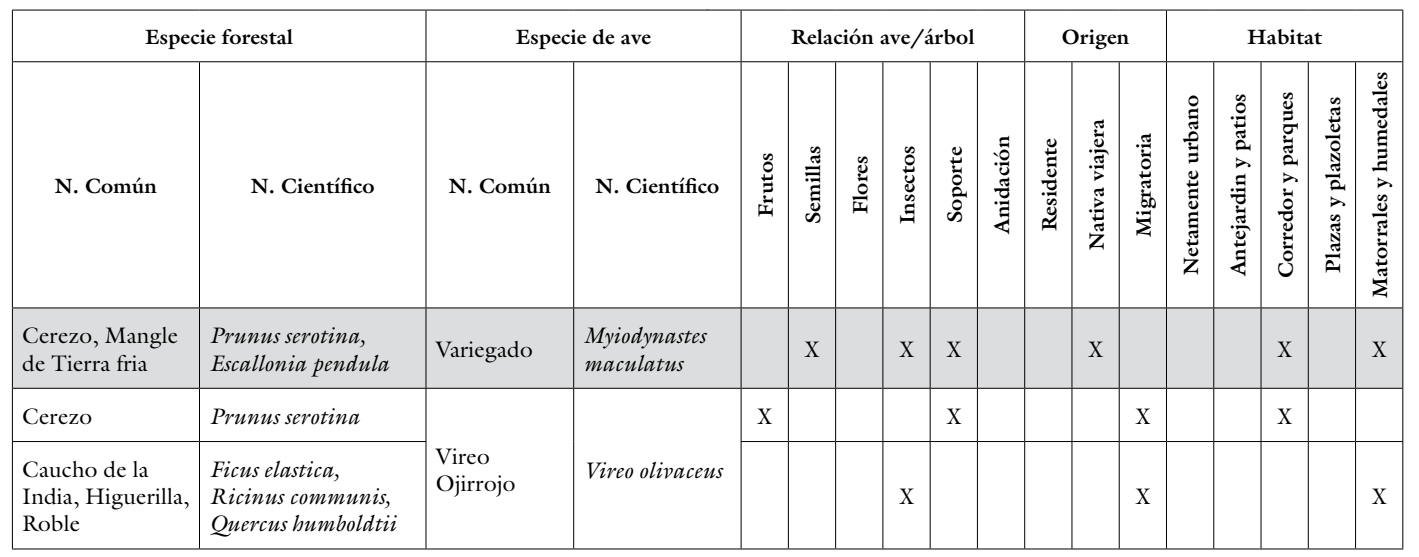

Fuente: elaboración propia con base en OpEPA y Bibliografía relacionada en este artículo.

\section{Discusión}

\subsection{Manejo de la avifauna en relación con la gestión del arbolado urbano}

En Bogotá la silvicultura urbana ha cobrado especial importancia debido a que el arbolado está siendo principalmente intervenido por el proceso de renovación urbana, por la construcción de su sistema de transporte público masivo y porque, además, en una proporción no despreciable, es una fuente de alto riesgo debido al mal estado físico y sanitario de muchos de sus individuos, causantes de los muchos y frecuentes accidentes por caída de ramas y por el volcamiento total de sus estructuras (Tovar, 2007).

Estas circunstancias han determinado el gran avance en la administración del arbolado urbano ubicado en espacio público, por medio de la autoridad ambiental de Bogotá y del Jardín Botánico, con un proyecto de manejo planificado, que ha permitido gestionar el censo georreferenciado de árboles urbanos más grande del continente, con un total de 1160526 individuos ubicados en el espacio público, tanto en el sistema de circulación (andenes), como en el sistema lúdico (parques), en el sistema hídrico (rondas de ríos y quebradas) y en las áreas de protección (humedales) (Tovar, 2013). El Decreto Distrital 531 de 2010 determina la obligatoriedad de tramitar un permiso para realizar cualquier tipo de tratamiento silvicultural en la ciudad, ello conlleva que el grupo de silvicultura urbana de la SDA reciba en promedio 300 solicitudes mensuales, que implica la evaluación técnica de una media de 2600 árboles al mes.

La tendencia de los permisos de tala en espacio público presenta un descenso continuo, de más de 20000 árboles en 2008, hasta estabilizarse alrededor de 4000 anuales desde 2013. En 2009 y 2010 se registró 
un alto número de autorizaciones debido a la adecuación del sistema de transporte masivo Transmilenio Fase III. Es muy probable que el número de autorizaciones aumente si se da vía libre a la construcción de las troncales Transmilenio de la Carrera 7, Avenidas Ciudad de Cali, Boyacá y 68 y la línea del metro elevado, en cumplimiento del Plan de Gobierno 2016-2019.

En predios privados, la tendencia de tala también ha bajado desde 2013, encontrándose la pérdida forestal en el rango entre 6000 y 8000 árboles año. De otra parte, la Unidad Administrativa Especial de Servicios Públicos (UAESP), durante el cuatrenio 2011-2015, realizó la poda de 90000 árboles promedio año, como parte del mantenimiento de la cobertura forestal (Tovar, 2016).

A pesar de la reducción de las intervenciones sobre el patrimonio forestal urbano, los individuos arbóreos presentes en las áreas de estudio tienen una altura promedio por debajo de los $10 \mathrm{~m}$, con DAP promedio inferior a los $40 \mathrm{~cm}$. Esto muestra cómo la comunidad vegetal asociada con los espacios verdes en la ciudad son principalmente comunidades jóvenes con un fuerte manejo que renueva permanentemente el arbolado de la ciudad. Ello genera efectos directos sobre las especies de aves presentes en tales coberturas. La tendencia en todas las ventanas muestra comunidades de aves altamente homogeneizadas, presentándose baja equitabilidad por la concentración de gran número de individuos en pocas especies. marcando con pintura amarilla los árboles ya revisados a lo largo del proyecto.

Para realizar intervenciones silviculturales apropiadamente, la ciudad cuenta con numerosos instrumentos técnicos como guías, manuales, lineamientos y protocolos. Uno de ellos es la Guía de Manejo Ambiental para el desarrollo de proyectos de infraestructura urbana en Bogotá D.C., Resolución 991 de 2001, que tiene en cuenta el manejo de la avifauna en relación con el manejo de los árboles que por objeto de la construcción de grandes obras en la ciudad tengan que ser talados o trasladados.

Esta guía se enfoca en las acciones que se deben priorizar en el manejo de comunidades de aves sujetas a perturbaciones derivadas de la intervención del arbolado urbano por razón de la construcción, estableciendo una serie de procedimientos que permiten que las empresas o entidades (públicas o privadas) encargadas de las obras puedan disminuir su impacto negativo sobre la avifauna local. Las actividades van desde el censado, rescate y relocalización de aves y nidos, crianza y liberación, pasando por las caracterizaciones de hábitat, hasta la socialización de este tipo de actividades a la comunidad.

Inicialmente, se requieren recorridos de búsqueda intensiva de nidos revisando árbol por árbol, para obtener el inventario de nidos. Luego el procedimiento determina el rescate de nidos, teniendo en cuenta el inventario y la programación de la ejecución de los tratamientos silviculturales, 
marcando con pintura amarilla los árboles ya revisados a lo largo del proyecto.

Durante la ejecución de las talas programadas, los rescates se deben realizar observando todas las normas de seguridad para el operario, el tráfico y las aves. Las nidadas rescatadas en buen estado, huevos y polluelos, deben ser trasladadas a las URRAS (Unidad de Rescate y Rehabilitación de Animales Silvestres) de la Universidad Nacional, encargada del cuidado adecuado, seguridad, y rehabilitación de los individuos hasta que lleguen a la adultez para proceder a la liberación.

La seguridad de las aves y los huevos rescatados se debe garantizar mediante un traslado seguro, en cajas con sustrato blando; un transporte rápido y la utilización de mallas bajo nidos ubicados sobre 8 metros de altura, de difícil acceso y cuando sea necesario.

En las URRAS un veterinario evalúa el estado de salud de las aves y verifica si los huevos tienen embrión, mediante ovoscopia, que consiste en observar cada huevo a trasluz en un cuarto oscuro. Si los huevos están en buen estado y con embrión, se pasan a una incubadora artificial, donde, si no eclosionan pasados 20 días de incubación, son desechados. Si los polluelos eclosionados no sobreviven o si los rescatados mueren durante la cría, deben ser incinerados y desechados.

Si se rescatan polluelos, deben ser criados hasta la etapa juvenil, se deben alimentar y mantener cerca de una fuente de calor, luego trasladarlos a un "animalario", que es un encierro cubierto, con piso de tierra y ramas secas, donde deben habituarse a alimentarse por sí solos y a ejercitar el vuelo con mínimo contacto humano. En caso de recibir aves con alguna enfermedad viral, como la viruela aviar, deben ser incinerados para evitar su propagación. Cuando los individuos lleguen a la edad adulta deben ser liberados, preferiblemente en las proximidades de la obra y su supervivencia debe ser monitoreada durante el siguiente mes (Consorcio Troncal NQS sur tramo 1, MHC., BVQi., 2007).

Eventualmente, es posible implementar la técnica de arribamiento, procedimiento que induce a los padres a seguir al nido con polluelos o huevos, para que continúen criando la nidada en una nueva ubicación, muy próxima a la inicial. Así, los padres originales o un ave nodriza continuarán con la incubación y cría, sin necesidad de hacerlo artificialmente, elevando la posibilidad de supervivencia. Cada rescate es reportado en un cuadro de registro con los datos de ubicación, tamaño de la nidada, especies arbórea y del ave, y anotaciones adicionales.

Existen pocos datos disponibles respecto de las mediciones directas e indirectas de las que hablan Donnelly \& Marzluff (2004). A nivel de guías de manejo de aves en áreas urbanizadas; Australia, Parsons (2007); San Francisco Planning Commission (2011); Toronto, Fatal Light Awareness Program (FLAP, 2012) y City of Vancouver (2014) se enfocan en mitigar la accidentalidad por colisión contra construcciones de vidrio. No fue posible establecer comparación con otras ciudades en 
términos de relaciones ave-árbol e impacto sobre la anidación por disturbios de origen humano, específicamente por construcción, ya que aun cuando existen las guías de manejo, como en Medellín, Moreno \& Hoyos (2015), no se encuentran en la literatura resultados de su aplicación.

Los parámetros que contiene la guía de manejo de aves en área urbana aplicada en Bogotá, respecto de censo, rescate, protocolo sanitario, crianza y liberación, corresponden con los utilizados a nivel internacional, detallados en Ralph J.C. et al. (1996) y Blair (1999), para el estado de California USA, en Sutherland et al. (2004), para el Reino Unido y para Bruselas y Barcelona, en Herrando et al. (2012). Sin embargo, algunos ornitólogos locales no están del todo de acuerdo con la aplicación de la guía actual; en los informes de ejecución de obras registra, que el rescate de nidos con huevos o neonatos es una tarea riesgosa para el operario e infructuosa en resultados, y además costosa porque algunas empresas se empeñan todavía en usar grúas de brazo con canastilla para todos los casos; también afirman que el "arribamiento", solo funciona en casos en que los polluelos puedan emitir alguna vocalización fuerte y la distancia sea muy próxima al origen, verificando que los padres lo ubiquen, situación que en las condiciones de una obra es de difícil práctica. Además, señalan que los centros de atención veterinaria todavía no han podido alcanzar niveles exitosos, en la incubación de huevos o crianza de neonatos hasta la etapa adulta para liberarlos.
Sería preferible, entonces, ubicar en el recorrido de inventario, nidos vacíos y removerlos antes de que sean usados nuevamente para anidar; ajustar el cronograma de obra para talar de último los árboles con nido activo, para esperar a que los huevos o neonatos se desarrollen hasta la formación de plumas; y solo en último caso, rescatar las nidadas con polluelos viables empleando cuerdas para escalar o escalera y malla de seguridad.

\subsection{Dualidad técnica en el manejo forestal urbano}

El manejo de la silvicultura urbana en Bogotá determina dos tipos de intervención que presentan diferencias ostensibles en objetivos, selección de especies, procedimientos técnicos y de mantenimiento, lo cual hace que sean excluyentes de acuerdo con el área objetivo; el precepto de arborización urbana cuya área de aplicación es tanto la cobertura blanda como la dura en área urbana; y el precepto de restauración ecológica cuya aplicación se circunscribe exclusivamente a las áreas protegidas de humedal, a los parques ecológicos de montaña, ubicados en área urbana y las rondas hídricas en área rural.

El precepto de arborización urbana tiene a su vez criterios ecológicos, como la integralidad, versatilidad, viabilidad, funcionalidad y eficiencia de la cobertura arbórea, atributos que buscan hacer de Bogotá una ciudad sostenible; criterios paisajísticos, como la armonía, proporción, estética, aspectos sonoros y sensoriales, 
que creen paisajes, a la vez que sean capaces de mimetizar, minimizar y contrastar la rigidez de las construcciones mediante la adición de espacios que representen la naturaleza dentro de la ciudad para satisfacer el gusto ciudadano por lo bello y lo agradable; criterios sociales que tienen que ver con la participación ciudadana en forma transversal en todas las fases de la construcción y transformación física de escenarios con el fin de promover y consolidar la malla verde de la ciudad; y criterios urbanísticos que se refieren a la respuesta de diseño frente a aspectos normativos y físicos que condicionan la ubicación de la arborización o de determinadas especies, y de seguridad para la ciudadanía, por la necesidad de mantener la transparencia y la visibilidad peatonal y vehicular Jardín Botánico de Bogotá (JBB, 2010). Estos últimos son los más restrictivos en términos de distanciamiento entre especímenes, restricción en los tipos de porte seleccionados, y en la generación de estructura de bosque urbano por la necesidad de transparencia en el sotobosque y en el dosel bajo.

Por el contrario, el precepto de restauración ecológica basa su intervención en objetivos de restablecimiento de dinámicas sucesionales, tomando como base a un ecosistema predisturbio (Cairns, 1993; Woodwell, 1994; Bradshaw, 1993) para reestablecer la estructura, el funcionamiento, la diversidad y las dinámicas del ecosistema específico (Aronson et al., 1993) para que este sea capaz de autosostenerse (Ehrenfeld \& Toth, 1997). Para ello, utiliza técnicas de restauración asistida o sucesión dirigida para generar condiciones que conlleven a procesos de recuperación, rehabilitación y conservación que limitan fuertemente la intervención antrópica de las áreas objeto.

La recuperación aborda el desarrollo de trabajos en sitios severamente degradados. No pretende restablecer la diversidad original, pero sí la función productiva o algunos de los servicios ecológicos originales (Lamb \& Gilmour, 2003). Entre tanto, la rehabilitación ecológica se orienta a asistir al ecosistema para el restablecimiento de elementos estructurales o funcionales que permitan reponer pequeños beneficios ecológicos de una restauración ecológica total (Clewell et al., 2000).

\subsection{Conectividad}

Las áreas naturales ubicadas en la mayoría de los casos en la periferia y con una muy fuerte presión de intervención se constituyen en el principal refugio para las especies que paulatinamente se han retirado de las áreas actualmente urbanizadas. Los cerros orientales, humedales, parques naturales y parques metropolitanos se constituyen en la última oportunidad para la conservación, que permita a algunas especies colonizar de nuevo algunas áreas dentro del contexto urbano.

Es clara la trascendencia del rol que cumplen los espacios lineales como los corredores de ronda, viales, férreos y canales y cursos de agua, como conectores entre los diferentes espacios verdes presentes en la ciudad desde las áreas naturales hasta los pequeños parques de bolsillo. Sin territorias 40

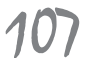


embargo, la planificación y conservación de la EEP y la promoción de una conectividad funcional aún no se consolidan como el eje de la política pública para el desarrollo ambiental de Bogotá. Tal conectividad es la de mayor interés para la comunidad de aves, ya que podría asegurar su movilidad y persistencia en la ciudad.

Terán (2009) evaluó la incidencia de variables de tipo urbano en la comunidad de aves en la ciudad y específicamente en la presencia o ausencia de algunas especies, mediante la caracterización y correlación de variables asociadas con el entorno urbano de cada punto evaluado en el estudio producto del contrato-convenio 046 de 2007 entre la SDA y ABO. De esta forma, definió categorías de configuración del paisaje basadas en la predominancia espacial de arbustos y rastrojos, pastizales y concreto. Igualmente, agrupó los puntos de observación en otros gradientes que podrían definir la matriz urbana como la densidad habitacional y el área que ocupan las vías principales y secundarias en un búfer de 200 $\mathrm{m}$. En los elementos asociados con áreas de uso público, tanto los de gran tamaño como parques metropolitanos y cementerios, como los de tamaño intermedio y pequeño como los parques de bolsillo, existe una clara homogeneización de las aves presentes, siendo las generalistas las que se favorecen de la degradación de las áreas verdes de la ciudad y su simplificación a coberturas como pastos y árboles dispersos.

Según Marzluff y Ewing (2001), se puede dar funcionalidad a los fragmentos generados en área urbana, por medio del enriquecimiento de la vegetación nativa; el manejo del paisaje que rodea al fragmento (matriz), no solo del fragmento; el aumento de la diversidad de la alturas del follaje dentro de las áreas fragmentadas; la integración de los parques urbanos por medio de corredores; la anticipación de la urbanización para reducir los efectos crecientes de la urbanización en zonas naturales perimetrales; sin embargo, es necesario aceptar que los fragmentos pueden ser los más adecuados para conservar solo unas pocas especies, por lo que se requiere estudiarlos y desarrollar programas de monitoreo que midan su aptitud.

La conectividad requiere de generación de más zonas verdes, por medio de la toma de decisiones del urbanismo que reconstruye la ciudad en su interior, desde la planificación y con una normatividad adecuada para tal fin. Desde la planificación, se debe buscar el enlace de las zonas verdes generadas en los planes parciales, las zonas de cesión de las nuevas urbanizaciones, la ampliación de las zonas de protección ambiental de las vías y servicios públicos para generar corredores verdes arborizables; y en donde no haya mayor posibilidad, el gobierno de la ciudad debería adquirir manzanas urbanizadas y demolerlas para generar parques de bolsillo y zonales, que además de cumplir la función de conectividad, puedan aproximar los pulmones verdes al lugar de residencia de los ciudadanos. Sin embargo, ello no es suficiente debido a que la generación de conectividad entre áreas verdes va más allá de una función estructural del paisaje o la creación de 
corredores lineales, ya que puede causarse un efecto adverso como el favorecimiento de la predominancia aún más marcada de las especies comunes. Por ello, es necesario revisar y planificar las especies vegetales que deben ser plantadas en estos espacios, pues deben tener la versatilidad de cumplir con la doble funcionalidad de ser hábitat de avifauna de especies poco comunes y raras, y ser especies aptas para el arbolado urbano.

La selección de especies arbóreas que brinden amplia oferta de alimento y refugio para las aves, y que busquen simultáneamente establecer condiciones para la colonización de nuevas especies de pájaros, debe constituirse en una directriz para los responsables de realizar los diseños paisajísticos de las nuevas obras civiles. Los nuevos arreglos florísticos pueden generar una EEP con mayor conectividad a nivel funcional y puede tener alcances importantes en el proceso de recolonización de especies de avifauna que permanecen en la periferia, asociadas con el mejor estado de conservación, para ello se requiere la identificación clara de rutas donde estas áreas puedan apoyar la movilidad de las especies de aves de mayor interés.

\subsection{Urbanismo}

De acuerdo con Müller y Werner (2010), la ciudad es el lugar donde la mayoría de las personas tienen contacto con la naturaleza; sin embargo, la expresión humana más común es la construcción de obras. En Bogotá existen algunos avances en materia de normatividad para la generación de espacio público, en este sentido, para la edificación de predios privados, el decreto Distrital 436 de 2006 establece que el determinante para la cesión mínima de espacio público para el desarrollo de un plan parcial es la mayor área entre el tope mínimo obligatorio de área verde, $4 \mathrm{~m}^{2}$ por habitante o el $17 \%$ del área neta urbanizable. Según el cálculo basado en la información contenida en el Boletín de Renovación Urbana (Cámara de Comercio de Bogotá, 2014), el promedio de $\mathrm{m}^{2}$ por habitante de área verde generado por el desarrollo de los planes parciales es de 4,87. Por tanto, es notable que, de acuerdo con el crecimiento de población, se presenta un gran déficit en la creación de área verde arborizable en la ciudad, ya que debiera ser de $9 \mathrm{~m}^{2}$ de área verde per cápita, según lo recomendado por la Organización Mundial de la Salud, para construir ciudades sostenibles.

En congruencia con lo anterior, es necesaria la modificación del denominado decreto de andenes (Dec. 561 de 2015), con el fin de incluir sistemas constructivos como el de andenes suspendidos que garanticen el espacio y la cantidad de sustrato mínimo vital para el buen arraigo de arbolado con el fin de evitar futuros volcamientos y otros como los puentes y los canales de raíces que eviten o minimicen daños del arbolado a la infraestructura. Asimismo, debería derogarse la Resolución conjunta de las Secretarías de Ambiente y Planeación 073 de 2017 que restringe casi totalmente la obligación de compensar las territarias 40 109 
áreas endurecidas y modificarse el artículo $7^{\circ}$ de la Resolución SDA 456 de 2014 , que reglamenta el Acuerdo Distrital 327 de 2008, norma que determinó que el endurecimiento de área verde por razón de la construcción de obra pública debería realizarse en área verde efectiva dentro del área de influencia del proyecto. En el mencionado artículo, la SDA determinó una escala para la ubicación de la compensación en relación con el concepto de área de influencia del proyecto así: endurecimiento superior a $5001 \mathrm{~m}^{2}$, el área de influencia es toda el área urbana del Distrito Capital; de 1001 a $5000 \mathrm{~m}^{2}$, es la cuenca y de 0 a $1000 \mathrm{~m}^{2}$, es el área de zonificación climática. Por supuesto, la mayor parte de las intervenciones de obra pública endurecen más de $5000 \mathrm{~m}^{2}$ teniendo la posibilidad de compensar dicha área en cualquier parte del área urbana de Bogotá, siendo lugares de compensación las zonas periféricas de la ciudad. Ello contraviene el sentido del Acuerdo Distrital y promueve el endurecimiento y la mayor fragmentación de las zonas verdes al interior de la ciudad (Tovar, 2016).

Con las prácticas de urbanismo actual se está generando la simplificación progresiva de la vegetación, la cual facilita la predominancia de especies comunes y generalistas en la ciudad. Es el caso del chamón (M. bonariensis), especie parásita de nidos y considerada una amenaza para las poblaciones de copetón (Z. capensis) y de chirriador o cucarachero endémico (Cistothorus apolinari), según Villaneda-

\section{tersitarias 40}

chado estos corredores para colonizar rápidamente algunos espacios de la ciudad, afectando así la posible recolonización y establecimiento de otras especies; por tanto, es importante analizar mejor la distribución de esta especie a lo largo de los elementos lineales, pues una posible medida de control consistiría en enriquecer la vegetación para evitar áreas conectadas de tipo pastizal.

\subsection{Identificación de algunas relaciones entre especies arbóreas y de avifauna}

Con 86012 individuos ubicados en el espacio público, según el sistema para la gestión del arbolado urbano (SIGAU, 2007), la especie más abundante en la ciudad es el sauco (S. peruviana), que, aunque no es nativa, es andina, y, además de formar sotobosque, es fuente de frutos para muchas especies de aves. Sin embargo, con el fin de dar mayor heterogeneidad al inventario forestal urbano, es necesario favorecer la plantación de especies que ofrezcan un potencial parecido como el jazmín del cabo (P. undulatum), tuno (Miconia squamulosa), tominejo (Palicourea spp), laurel (Myrica parvifolia), arrayán (Myrciantes leucoxyla), té de Bogotá (Symplocos theiformis) y granizo (Hedyosmun bonplandianum).

Debido al uso extensivo que las aves hacen del follaje buscando alimento, principalmente de insectos e invertebrados pequeños, es importante contar con especies frondosas, como los cauchos nativos 
(Ficusspp), y tominejeros (Pallicourea spp o Faramea spp).

Es importante, además, contar con especies vegetales de gran fuste, que atraigan a sus copas diversos insectos y produzcan frutos que sirvan de alimento para colibríes, golondrinas y aves frugívoras. Dentro de estas especies, puede contarse con el pino romerón (Decussocarpus rospigliossi), el gaque o cucharo (Clusia multiflora), caucho sabanero (Ficus soatensis) y otras especies de media altura como el siete cueros (Tibouchina lepidota), y el juco o garrocho (Viburnum triphillum). También arbustos como el chilco (Bacharis bogotensis), el pegamosco (Befaria resinosa) y la escalonia (Escallonia paniculata).

El comportamiento territorial de la mirla (T. fuscater) restringe la presencia de otras aves que pudieran colonizar sectores de área urbana. Esta situación puede ser susceptible de manejo mediante el control de su principal fuente de alimento, el fruto del cerezo. La presencia de este árbol en la ciudad registró 15430 árboles (SIGAU, 2007), su manejo ha determinado la plantación de 416, (SIGAU, 2016) y la autorización de tala de 443 individuos, registrados en el Sistema de Información Ambiental (SIA, 2016). Sin embargo, la decisión de restringirlo afectaría otras especies que tienen estrecha relación con él como: sirirí norteño (Tyrannus tyrannus), cotinga (Ampelion rubrocristatus), verderón (Vireo olivaceus), buchipechosa o zorzal (Catharus ustulatus), azulejo (Thraupis episcopus), clarinero (Anisognathus igniventris), picogordo (Pheucticus aureoventris) y los cardenales (Piranga rubra) y (Piranga olivacea) (OpEPA, 2017).

En contraposición de las situaciones adversas que genera el eucalipto (Eucalyptus sp), como su excesivo consumo de agua, su efecto sobre la química del suelo (FAO, 1987), los daños a la infraestructura vial y peatonal (Beltrán, 2011; Fernández, 2013) y el riesgo que supone por su susceptibilidad al volcamiento, esta especie, en relación con las aves, presenta bondades no reconocidas como la de ser melífera (Zerda-Ordoñez, 1994) y, por tanto, fuente de alimento principalmente para los toches (I. chrysater) y colibríes de los géneros Colibri, Lesbia, Ramphomicron, Metallura, Eriocnemis, Pterophanes, Chaetocercus y Ensifera. Sus semillas son consumidas por la torcaza (Z.auriculata) y el jilguero (Carduelis spinescens) (OpEPA, 2017). Y su atracción de insectos, sumado a lo anterior, convierten a la especie en medio de generación de alimento para las aves insectívoras. De otro lado, su enorme porte es esencial en la función de percha para las aves rapaces desde donde pueden ubicar su alimento sobre la superficie terrestre; por ello, aun cuando su presencia en andenes y separadores viales es riesgosa, es deseable en sectores restringidos de parques y humedales. El censo del arbolado urbano registró 37605 árboles de diferentes especies de eucalipto (SIGAU, 2007), a 2015 no se han vuelto a plantar en área urbana y por el contrario se han autorizado talar 1781 individuos (SIA, 2016). 


\section{Conclusiones}

La intervención que los proyectos de construcción tienen sobre el proceso de anidación de la avifauna es muy bajo, ya que tan solo en el 4,5\% de los árboles intervenidos se encontraron huevos y polluelos, por tanto su impacto es mínimo. Además, el manejo de avifauna centrado en la ubicación y rescate de nidos es costoso y solo beneficia a nueve especies comunes, que anidan y emplean los recursos de los corredores viales que se intervienen con las obras.

De otro lado, el impacto generado por las construcciones sobre la avifauna en términos de hábitat, movilidad, alimento y soporte es muy alto, ya que promueve el endurecimiento y la mayor fragmentación de las zonas verdes al interior de la ciudad, así como la simplificación progresiva de la vegetación, lo que facilita la predominancia de especies comunes y generalistas.

Con apego a los lineamientos para el manejo de la cobertura arbórea de Bogotá, y teniendo en cuenta la extensión y ubicación de las áreas donde deben aplicarse los preceptos de arborización urbana para constituir parches en favor de la conectividad de los espacios verdes, lo que debiera ser imperativo, así como el comportamiento y los requerimientos naturales de las aves, se puede inferir que las especies que tienen mayores oportunidades de colonizar nuevamente áreas dentro de la ciudad y que pueden responder rápidamente a acciones de enriquecimiento, tanto en composición como en estructura son: turpial andino o chisga (C. spinescens), gavilán maromero (Elanus leucurus), azulejo común (Thraupis episcopus), cometa coliverde (Lesbia nuna), oriol o turpial amarillo (Icterus nigrogularis), cometa colinegro (Lesbia victoriae) y el picogordo pechinegro (Pheucticus aureoventris). Para lograrlo, es necesario incluir en los diseños de arborización las especies vegetales directamente relacionadas con estas aves, que pueden consultarse en la tabla 4 .

\section{Referencias}

Adams, L.W. (2005). Urban wildlife ecology and conservation: A brief history of the discipline. Urban Ecosystems, 8, 139-156.

Alcaldía mayor de Bogotá, Instituto de Desarrollo Urbano (2005). Guía de Manejo Ambiental para el desarrollo de proyectos de Infraestructura urbana en Bogotá D.C. Recuperado de http://ambientebogota.gov.co/documents/664482/0/GUIA_MANEJO_AMBIENTAL_FINAL.pdf

Alcaldía mayor de Bogotá, Decreto 190 de 2004. Por medio del cual se compilan las disposiciones contenidas en los Decretos Distritales 619 de 2000 y 469 de 2003. Registro Distrital 3122 (22 junio 2004).

Alcaldía mayor de Bogotá, Decreto Distrital 436 de 2006. Por el cual se dictan disposiciones comunes a los planes parciales en tratamiento de desarrollo, y se establece la metodología para el reparto territarias 40 112 
equitativo de cargas y beneficios. Registro Distrital 3636. (19 octubre 2006).

Alcaldía mayor de Bogotá, Decreto 531 de 2010. Por el cual se reglamenta la silvicultura urbana, zonas verdes y la jardinería en Bogotá y se definen las responsabilidades de las Entidades Distritales en relación con el tema y se dictan otras disposiciones. Registro Distrital 4566. (24 diciembre 2010).

Alcaldía mayor de Bogotá, Decreto 561 de 2015. Por el cual se actualiza la Cartilla de Andenes, adoptada mediante Decreto Distrital 1003 de 2000, adicionada mediante el Decreto Distrital 379 de 2002 y actualizada mediante el Decreto Distrital 602 de 2007, y se dictan otras disposiciones. Registro Distrital 5740. (23 diciembre 2015).

Aronson, J., Floret, C., Le Floc'h., E., Ovalle, C., \& Pontanier R. (1993). Restoration and rehabilitation of degraded ecosystems in arid and semiarid regions. I. A view from the south. Restoration Ecology, 1(1), 8-17.

Barrera, J., \& Ríos, H. (2002). Acercamiento a la ecología de la restauración. PérezArbelaezia, 13, 33-46.

Bezzel, E. (1985). Birdlife in intensively used rural and urban environments. Ornis Fennica, 62, 90-95.

Beltrán, L. (2011). Evaluación de daños producidos por árboles ornamentales en pavimentos de la zona norte de Bogotá. Ingeniería e Investigación, 2(1982), 46-57.

Blair, R. (1999). Birds and butterflies along an urban gradient: surrogate taxa for assessing biodiversity? Ecological Applications, 9(1), 164-170.

Blair, R. (2001). Creating a homogeneous avifauna. En J. M. Marzluff, R. Bowman, \& R. Donnelly, (eds.), Avian ecology and conservation in an urbanizing world. (pp. 459-486). Norwell, Massachusetts: Kluwer Academic Publishers.

Bradshaw, A. D. (1993). Restoration ecology as a science. Restoration Ecology, 1,71-73.

Cámara de Comercio de Bogotá (2014). Boletín de Renovación Urbana en Bogotá. Bogotá: Cámara de Comercio de Bogotá.

Cano M. (2011). Informe final de manejo de avifauna. Expedientes SDA-03-09-61, SDA 03-09-480 y SDA 03-09-480 IDU Contrato 137 de 2007. Adecuación de la troncal Calle 26 al Sistema Transmilenio tramos 3 y 4 comprendidos entre la Transversal 76 y la Carrera 19. Inédito. City of Vancouver (2014). Bird friendly design guidelines explanatory note. Recuperado de https://vancouver.ca/ files/cov/bird-friendly-strategy-designguidelines-draft-2014-09-01.pdf

Clewell, A. F., Kelly J. P., \& Coultas C. L. (2000). Forest restoration at dogleg branch on phosphate-mined and reclaimed land, Florida. En W. L. Daniels y S.G. Richardson Proceedings of the 17th annual national meeting, Tampa, Flori$d a($ pp.197-204). Lexington, Kentucky: American Society for Surface Mining and Reclamation.

Concejo de Bogotá. Acuerdo Distrital 327 de 2008. Por medio cual se dictan normas territarias 40

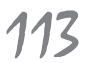




\section{tersitarias 40} 114 para la planeación, generación y sostenimiento de zonas verdes denominadas "Pulmones Verdes" en el Distrito Capital y se dictan otras disposiciones (24 Septiembre 2008).

Consorcio Troncal NQS sur tramo 1, MHC., BVQi. (2007). Balance manejo de avifauna. Contrato IDU 179/03. Adecuación de la Troncal NQS Sector Sur, al sistema Transmilenio Tramo: Calle 10 - Escuela de policía General Santander. Delgado, C., \& Correa J. (2013). Estudios ornitológicos urbanos en Colombia: Revisión de literatura. Ingeniería y Ciencia, 226, 215-236.

Donnelly, R. E., \& Marzluff, J. M. (2004). Designing research to advance the management of bird in urbanizing areas. En Shaw et al., Proccedings $4^{\circ}$ International Urban Symposium (pp. 114-122). Arizona: W. Shaw and L Harris. Eds.

Ehrenfeld, J. G., \& Toth, L. A. (1997). Restoration ecology and the ecosystem perspective. Restoration Ecology, 5, 307-317.

Erz, W. (1966). Ecological principles in the urbanization of birds. Ostrich Supplement, 6, 357-363.

European Commission (2013). Building a Green infrastructure for Europe, 7, 24. Luxemburg: Publications Office of the European Union.

FAO. Poore, M. E., \& Fries, C. (1987). Efectos ecológicos del eucalipto. Roma: Organización de las Naciones Unidas para la Agricultura y la Alimentación.

Fernández, M. A. (2013). Relaciones entre las especies arbóreas, sus características morfológicas y los tipos de suelo, y las afectaciones en la infraestructura vial de la ciudad de Bogotá (Trabajo grado, Pontificia Universidad Javeriana, Bogotá, Colombia).

FLAP Fatal Light Awareness Program (2012). Volunteer Training Manual. Bird rescue, First aid, transport, release. Toronto: Wildlife Centre.

Garrido, L. (2007). Informe final de manejo de avifauna. Expediente SDA-DM 032007-1832. Contrato IDU 095 de 2006. Construcción de andenes y cicloruta de la avenida Agoberto Mejía entre las calles 53 y 13 sur. Inédito.

Herrando, S., Weiserbs, A., Quesada, J., Ferrer X., \& Paquet, J. Y. (2012). Development of urban bird indicators using data from minitoring schemes in two large European cities. Animal Biodiversity and Conservation, 35(1), 141-150.

Jardín Botánico de Bogotá (2010). Manual de Silvicultura Urbana para Bogotá. Alcaldía Mayor de Bogotá D.C. Bogotá: Imprenta Nacional de Colombia.

Knignt, R. L. (1990). Ecological principles applicable to the management of urban ecosystems. Perspectives in urban ecology. Denver, Colorado: Museum of Natural History and Thorne Ecological Institute.

Lamb, D., \& Gilmour, D. (2003). Rehabilitation and restoration of degraded forests. Gland, Switzerland: IUCN, Gland, Switzerland and Cambridge, UK and wwF, The World Conservation Union.

León, M. (2007). Informe final de manejo de avifauna. Expediente SDA 03-07-1926. 
Contrato IDU 015 de 2006. Construcción de dos pontones sobre el canal Salitre y rio Juan Amarillo acceso al barrio Lisboa, localidad de Suba. Inédito.

Marzluff, J. M., Gehlbach F. R., \& Manuwal D. A. (1998). Urban environments influences on avifauna and challenges for the avian conservationist. En J. M. Marzluff \& R. Sallabanks (eds.) Avian Conservation: research and management (pp. 283-299). Washington D.C.: Island Press.

Marzluff, J. M. (2001). Worldwide urbanization and its effects on birds. En En J. M. Marzluff \& R. Sallabanks (eds.) Avian conservation and ecology in an urbanizing world (pp. 19-47). Washington D.C.: Island Press.

Marzluff, J. M., \& Ewing, K. (2001). Restoration of fragmented landscapes for the conservation of birds: A general framework and specific recommendations for urbanizing landscapes. Restoration Ecology, 9(3), 280-292.

McCallum, H., \& Dobson, A. (2002). Disease, habitat fragmentation and conservation. The Royal Society, 269, 20412049.

Molina, J. (2006). Informe final de manejo de avifauna. Expedientes SDA-DAMA-DM 07-99-023; DM 03-2004-1398 y DM 0305-1192 Transmilenio. Contrato IDU 180 de 2003. Adecuación de la Troncal NQS al sistema Transmilenio tramo 2 sur, comprendido la Escuela de policía General Santander y la Avenida ciudad de Villavicencio en Bogotá D.C. Inédito.
Moreno, F., \& Hoyos, C. (2015). Guía para el manejo del arbolado urbano en el Valle de Aburrá. Medellín: Área Metropolitana del Valle de Aburrá \& Universidad Nacional de Colombia.

Muller, N., \& Werner, P. (2010). Urban Biodiversity and the case for implementing the convention on biological diversity in towns and cities. En N. Muller, P. Werner, \& J.G. Kelcey (eds.) Urban biodiversity and desing (pp. 1-33). London: Conservation Science and Practice Series. Wiley-Blackwell and the Zoological Society of London.

Parsons, H. (2007). Best practice guidelines for enhancing urban bird hábitat. Birds in backyard program. Australia: Birds Sydney Australia.

Ralph, J. C., Geupel, G. R., Pyle, P., Martin, T. E., DeSante D. F., \& Millá, B. (1996). Manual de métodos de campo para el monitoreo de aves terrestres. General Technical Report PSW-GTR-159. Albany, CA: Pacific Southwest Research Station, Forest Service, U.S. Department of Agriculture.

Ramírez, C. (2004). Informe final de manejo de avifauna. Expediente SDA-DAMA-DM 03-07-2809. Contrato IDU 145 de 2003. Adecuación de la troncal Avenida Suba al Sistema Transmilenio tramo 1 comprendido entre la Avenida Ciudad de Cali y el Portal de Suba. Inédito.

Ramírez, C. (2004). Informe final de manejo de avifauna. Expediente SDA-DAMADM-07-99-23. Contrato IDU 146 de 2003. Adecuación de la troncal Avenida Suba al Sistema Transmilenio tramo 2 


\section{territarias 40} 116 comprendido entre la Calle 127 y la Avenida Ciudad de Cali. Inédito.

Remolina, F. (2006). Propuesta de tipología de corredores para la Estructura Ecológica Principal de Bogotá, Revista nodo, 1(15), 13-20.

San Francisco Planning Commission (2011). Standards for bird-safe buildings. San Francisco: San Francisco Planning Department.

SDA-ABO. (2009). Informe Técnico Final de Análisis Convenio 046 de 2007 entre la Secretaría Distrital de Ambiente y la Asociación Bogotana de Ornitología. Criterios técnicos de conectividad ecológica con énfasis en la conservación de la avifauna y la consolidación de procesos de restauración en la estructura ecológica principal del Distrito Capital. Inédito.

SDA-DAMA. Resolución 991 de 2001. Por la cual se adopta para el Distrito Capital de Bogotá, la "guía de manejo ambiental", para el desarrollo de proyectos de infraestructura urbana que causen mínimo impacto ambiental. Legislación económica 1175. (30 septiembre 2001).

SDA-DAMA Expediente DM 03-2006-1687. Contrato IDU 145 de 2003. Adecuación de la troncal Avenida Suba al Sistema Transmilenio tramo 2 comprendido entre la Calle 80 y la Calle 127. Informe final de manejo de avifauna. Inédito.

SDA-DAMA Expediente DM 03-23-99 Transmilenio. Contrato IDU 179 de 2003. Adecuación de la Troncal NQS al sistema Transmilenio tramo 1 sur, comprendido entre la Calle 10 y la Escuela de policía
General Santander. Informe final de manejo de avifauna. Inédito.

SDA-DAMA Expediente DM-03-03-1330. Contrato IDU 106 de 2003. Troncal NQS tramo 2 norte, entre la avenida paseo los libertadores (Calle 92) y la avenida Gabriel Andrade Lleras (Calle 68). Informe final de manejo de avifauna. Inédito.

SDA Expediente DM 03-06-1835. Contrato IDU 106 de 2003. Adecuación al sistema Transmilenio de la Troncal NQS, sector Norte, Tramo 1, comprendido entre la Calle 10 y la Calle 6. Informe final de manejo de avifauna. Iinédito.

SDA Expediente 03-2007-1324. Contrato IDU 128 de 2006. Intervenciones integrales de la malla vial local de grupo vial fase 1, grupo 2, localidad de Suba. Informe final de manejo de avifauna. Inédito.

SDA Expediente DM 03-08-3909. Contrato IDU BM 237 de 2004. Construcción y/o rehabilitación de vías de rutas alimentadoras del sistema Transmilenio, zona 4, grupo 1. Informe final de manejo de avifauna. Inédito.

SDA Expediente DM 03-07-1918. Contrato IDU 178 de 2006. Construcción y/o rehabilitación de vías en varias localidades, grupo 2 -localidades de Usme, Mártires, Santafé y Rafael Uribe-. Informe final de manejo de avifauna. Inédito.

SDA Expediente DM 03-2007-1749. Contrato IDU 179 de 2006. Construcción y/o rehabilitación de vías en varias localidades, grupo3, localidad Kennedy. 
Informe final de manejo de avifauna. Inédito.

SDA Expediente SDA-03-2009-1642. Contrato IDU 124 de 2006. Intervenciones integrales a la malla vial local grupo vial fase 3 grupo 2, localidades Tunjuelito y Ciudad Bolívar. Informe final de manejo de avifauna. Inédito.

SDA Expediente GTS913 29-06-07. EAAB. 1-01-25500-676-2006 Obras de canalización del río Fucha, interceptor izquierdo del Fucha, pondaje y obras anexas, sector pondaje y sector Avenida Boyacá-campamento. Informe final de manejo de avifauna. Inédito.

SDA Expediente DM 03-06-1835. Contrato IDU 116 de 2005. Ampliación y rehabilitación de la autopista vía al llano entre el CAI Yomasa y el inicio de la concesión Bogotá-Villavicencio en el tramo comprendido entre el $\mathrm{Kl}+575$ al $\mathrm{K} 5+324$. Informe final de manejo de avifauna. Inédito.

SDA Expedientes DM-03-2004-1653 y DM 03-2006-2467 IDU Contratos 106 de 2005 y 154 de 2006. Construcción de la Avenida Ciudad de Cali desde la Transversal 91 hasta el aproche oriental de los puentes vehiculares sobre el brazo del Humedal Juan Amarillo en Bogotá. Informe final de manejo de avifauna. Inédito.

SDA. Resolución 456 de 2014. Por medio de la cual se establecen los lineamientos y procedimientos para la compensación por endurecimiento de zonas verdes por desarrollo de obras de Infraestructura. Registro Distrital 5297. (12 febrero 2014).

Sutherland, W., Newton I., \& Green R. (2004). Bird Ecology and Conservation. A Handbook of techniques. Oxford: Oxford University Press.

Terán, P. (2009). Estudio del impacto de variables del paisaje urbano en la composición y estructura de la avifauna de la ciudad de Bogotá. (Trabajo grado, Pontificia Universidad Javeriana, Bogotá, Colombia).

Tovar, G. (2007). Manejo del arbolado urbano en Bogotá. Territorios, 16 y 17, 149-173.

Tovar, G. (2013). Aproximación a la Silvicultura Urbana en Colombia. Bitácora Urbana, 22, 119-136.

Tovar, G. (2016). Propuesta de plan para la gestión de la infraestructura verde urbana de Bogotá Distrito Capital. (Trabajo de Maestría, Pontificia Universidad Javeriana, Bogotá, Colombia).

Villaneda-Rey, M., \& Rosselli, L. (2011). Abundancia del Chamón Parásito (Molothrus bonariensis, Icteridae) en 19 humedales de la Sabana de Bogotá, Colombia. Ornitología Colombiana, 11, 37-48.

Zerda-Ordoñez, E. (1994) Historia Natural del Tominejo Colibri coruscans coruscans (Gould) (Aves, Trochilidae). Universitas Scientiarium, 2(1), 65-85. 
